\title{
Isotopic exchange on the diurnal scale between near-surface snow and lower atmospheric water vapor at Kohnen station, East Antarctica
}

\author{
François Ritter ${ }^{1,2}$, Hans Christian Steen-Larsen ${ }^{2,3}$, Martin Werner ${ }^{1}$, Valérie Masson-Delmotte ${ }^{2}$, Anais Orsi $^{2}$, \\ Melanie Behrens ${ }^{1}$, Gerit Birnbaum ${ }^{1}$, Johannes Freitag ${ }^{1}$, Camille Risi ${ }^{4}$, and Sepp Kipfstuhl ${ }^{1}$ \\ ${ }^{1}$ Alfred-Wegener-Institut (AWI) Helmholtz Zentrum für Polar und Meeresforschung, Bremerhaven, Germany \\ ${ }^{2}$ Laboratoire des Sciences du Climat et de l'Environnement (LSCE), IPSL/CEA-CNRS-UVSQ, \\ Saclay, Gif-sur-Yvette, France \\ ${ }^{3}$ Centre for Ice and Climate, Niels Bohr Institute, University of Copenhagen, Copenhagen, Denmark \\ ${ }^{4}$ Laboratoire de Météorologie Dynamique (LMD), IPSL/CNRS-UPMC, Paris, France
}

Correspondence to: François Ritter (fritte2@uic.edu)

Received: 12 January 2016 - Published in The Cryosphere Discuss.: 22 February 2016

Revised: 28 June 2016 - Accepted: 1 July 2016 - Published: 29 July 2016

\begin{abstract}
Quantifying the magnitude of post-depositional processes affecting the isotopic composition of surface snow is essential for a more accurate interpretation of ice core data. To achieve this, high temporal resolution measurements of both lower atmospheric water vapor and surface snow isotopic composition are required. This study presents continuous measurements of water vapor isotopes performed in East Antarctica (Kohnen station) from December 2013 to January 2014 using a laser spectrometer. Observations have been compared with the outputs of two atmospheric general circulation models (AGCMs) equipped with water vapor isotopes: ECHAM5-wiso and LMDZ5Aiso. During our monitoring period, the signals in the $2 \mathrm{~m}$ air temperature $T$, humidity mixing ratio $q$ and both water vapor isotopes $\delta \mathrm{D}$ and $\delta^{18} \mathrm{O}$ are dominated by the presence of diurnal cycles. Both AGCMs simulate similar diurnal cycles with a mean amplitude 30 to $70 \%$ lower than observed, possibly due to an incorrect simulation of the surface energy balance and the boundary layer dynamics. In parallel, snow surface samples were collected each hour over $35 \mathrm{~h}$, with a sampling depth of $2-5 \mathrm{~mm}$. A diurnal cycle in the isotopic composition of the snow surface is observed in phase with the water vapor, reaching a peak-to-peak amplitude of $3 \%$ for $\delta \mathrm{D}$ over $24 \mathrm{~h}$ (compared to $36 \%$ for $\delta \mathrm{D}$ in the water vapor). A simple box
\end{abstract}

model treated as a closed system has been developed to study the exchange of water molecules between an air and a snow reservoir. In the vapor, the box model simulations show too much isotopic depletion compared to the observations. Mixing with other sources (advection, free troposphere) has to be included in order to fit the observations. At the snow surface, the simulated isotopic values are close to the observations with a snow reservoir of $\sim 5 \mathrm{~mm}$ depth (range of the snow sample depth). Our analysis suggests that fractionation occurs during sublimation and that vapor-snow exchanges can no longer be considered insignificant for the isotopic composition of near-surface snow in polar regions.

\section{Introduction}

Thanks to the design of mass spectrometers and their application to stable water isotopes since the 1950 s, precipitation has long been sampled for laboratory stable isotope analyses in order to trace atmospheric processes related to the hydrological cycle (e.g., Dansgaard, 1964). Past changes in precipitation isotopic composition have also been investigated using a variety of natural archives. Among these, ice cores form one of the most direct records of the isotopic compo- 
sition of past precipitation. In Antarctica, stable water isotope measurements are central for past climate reconstructions from these ice cores, through atmospheric distillation processes connecting temperature, condensation and isotopic composition (e.g., Masson-Delmotte et al., 2008). However, the relationship between precipitation isotopic composition and climate is complex, as it is affected by fractionation taking place at most phase transitions, at evaporation, during atmospheric transport, and at condensation.

While spatial relationships have been documented using surface snow data (Masson-Delmotte et al., 2008), only a few studies have examined the drivers of Antarctic precipitation isotopic composition variability at timescales associated with synoptic or diurnal events (Fujita and Abe, 2006; Schlosser et al., 2004). In this study we focus on these timescales. The climatic controls on precipitation isotopic composition have been investigated under different climatic contexts thanks to the implementation of stable water isotopes into atmospheric general circulation models (AGCMs) (e.g., Joussaume et al., 1984). Classically, the mean isotopic composition of precipitation simulated by atmospheric models is directly compared to ice core data, thereby ignoring potential post-depositional processes which may transform the initial precipitation signal in the upper part of the firn. In this study we aim to investigate one such post-depositional process: the isotopic exchange between atmospheric water vapor and snow.

The possibility to monitor isotopic exchanges between surface snow and low-level atmospheric water vapor has emerged thanks to recent technological development. New laser spectrometers have been released in the 2000 s, creating a substantial advance within the field of water isotope research. These analyzers are able to perform continuous and in situ measurements of the humidity mixing ratio (defined as the mass of water vapor divided by the mass of dry air) and the stable water vapor isotope concentrations. Prior to this advance, water vapor could only be collected using tedious cold trapping methods (e.g., Jacob and Sonntag, 1991; Steen-Larsen et al., 2011; Angert et al., 2008), deployed until sufficient amounts were collected to allow subsequent transfer to vials and later mass spectrometer analyses. Such a task was almost impossible to perform routinely (e.g., Schwarz et al., 1998).

The first implementation of continuous in situ isotopic monitoring of surface water vapor isotopic composition above an ice sheet was achieved at NEEM, NW Greenland, during summer field seasons 2010-2012 (Steen-Larsen et al., 2013, 2014). In parallel, repeated sampling of surface snow was also implemented for laboratory isotopic analyses. The combined snow and vapor datasets revealed changes in the isotopic composition of surface snow in response to synoptic changes in the atmospheric water vapor isotope values and exhibited a strong diurnal variability in near-surface water vapor isotopic composition. Over several days and weeks, parallel variations between the surface snow isotopic composition and the near-surface vapor isotopic composition were identified in between snowfall events. This was surprising, as the snow isotopic composition was expected to be controlled by the isotopic composition of precipitation. The variations were interpreted to reflect interactions between the snow surface and the lower atmosphere occurring during surface snow metamorphism. This new finding has potential implications for the interpretation of ice core records and for the comparison of ice core data with atmospheric model results. It is unclear, however, whether surface air-snow exchanges could lead to significant changes in the snow isotopic composition at colder sites, such as those of the Antarctic Plateau.

Here, we report surface vapor isotope measurements performed above the Antarctic ice sheet. These measurements were performed at the German Kohnen station, a deep ice coring site with intermediate temperature and a humidity mixing ratio high enough in the summer to make accurate measurements of the water vapor isotopic composition. In parallel, snow surface samples were collected for comparison with the vapor on a diurnal scale. The surface of the ice sheet around Kohnen is characterized by the presence of large sastrugi, created by wind redistribution and sublimation of snow, hence producing considerable variability in the snow surface age, origin and density. In particular, very hard dunes sticking up above the mean surface level may be half a year old (Birnbaum et al., 2010). A previous study performed at Vostok station (Antarctica) reported a large variability in the isotopic composition of the snow surface $(10 \mathrm{~cm}$ depth) over an $1 \mathrm{~km}$ transect, with a maximum variation of $30 \%$ in $\delta$ D over $100 \mathrm{~m}$ horizontally (Ekaykin et al., 2002). The isotopic composition of the snow surface at Kohnen station is expected to show a similar spatial variability, depending on the age and the origin of the snow patch. The importance of the sublimation and condensation processes in the isotopic composition of different snow patches will be investigated. The term "condensation" (rather than "deposition") is preferred in this paper to describe the water phase change from vapor to solid in order to avoid a possible confusion with "post-depositional processes".

After a brief overview of the Kohnen station environment (Sect. 2), this article details the successful implementation of continuous measurements of water vapor isotopic composition during the months of December 2013 and January 2014. Section 3 (material and methods) describes our protocol for water vapor data processing and reports the accuracy of the data. We also report the parallel surface snow sampling over $35 \mathrm{~h}$ and introduce simulations performed with two AGCMs equipped with stable water isotopes, LMDZ5Aiso and ECHAM5-wiso. In Sect. 4, we present observed and simulated values, first for the day-to-day variability and then for the diurnal cycles. We compare the diurnal variability of the isotopic composition of the water vapor and of the very first layer of surface snow through a box model. The last section summarizes our key conclusions and perspectives. 


\section{Kohnen station environment}

The German Kohnen station $\left(75^{\circ} 00^{\prime} \mathrm{S}, 0^{\circ} 04^{\prime} \mathrm{E}\right)$ is located on the Antarctic Plateau in Dronning Maud Land, $550 \mathrm{~km}$ from the South Atlantic coast line and $2892 \mathrm{~m}$ a.s.l. (above sea level). Near the station, the surface elevation has a gentle slope of $\sim 1.3 \pm 0.3 \mathrm{~m} \mathrm{~km}^{-1}$ with a direction of $\sim 61^{\circ}$. This place is characterized by katabatic winds $\left(\sim 8 \mathrm{~m} \mathrm{~s}^{-1}\right)$ on a diurnal timescale, which form around 03:00 UTC and vanish around 15:00 UTC (Van As et al., 2005). The katabatic regime can be interrupted by the influence of synoptic systems, responsible for $20 \%$ of snowfall events at Kohnen station (Schlosser et al., 2010). From December 2013 to January 2014, five snowfall events were observed and no significant snow accumulation was detected from daily measurements of snow surface height within a precision of $\sim 1 \mathrm{~mm}$ (protocol described in Sect. 3.5).

The mean temperature for the month of January 2014 was $-25^{\circ} \mathrm{C}$, similar to the climatological average $\left(-25 \pm 2{ }^{\circ} \mathrm{C}\right.$, 1998 to 2013). Both temperature values are based on hourly data from the permanent automatic weather station (AWS) at Kohnen (hereafter AWS9, described in Sect. 3.1). During clear-sky conditions, the net radiation at the surface is predominantly positive during the day (i.e., a dominant energy gain by shortwave radiation from the sun despite a high albedo of the snow surface) and negative during the night (i.e., a net loss by longwave emissions from the surface), imprinting a strong diurnal cycle to the surface temperature, with a peak-to-peak amplitude of $14{ }^{\circ} \mathrm{C}$ (Van As et al., 2005).

\section{Material and methods}

\subsection{Weather observations}

Weather observations are reported every hour and precipitation events are labeled "snowfall", "light snowfall" or "diamond dust". A snowfall event leaves a visible accumulation on flat surfaces (for example transport boxes), whereas during a light snowfall event or a diamond dust event no visible accumulation is observed. During the time of observations, snowfall and light snowfall events were almost always associated with $6 / 8$ to $8 / 8$ cover of low-level clouds whereas the cloud cover of low-level clouds was almost always $0 / 8$ to $2 / 8$ in case of diamond dust.

Two AWSs were also installed at Kohnen (Fig. 1). AWS9 is permanent and has performed measurements since $\sim 1998$. AWS 13/14 was only temporarily installed at Kohnen for the summer season, from December 2013 to January 2014. Hourly averages of the following parameters measured at $2 \mathrm{~m}$ height are available: air pressure, temperature, relative humidity $(\mathrm{RH})$, wind speed and wind direction.

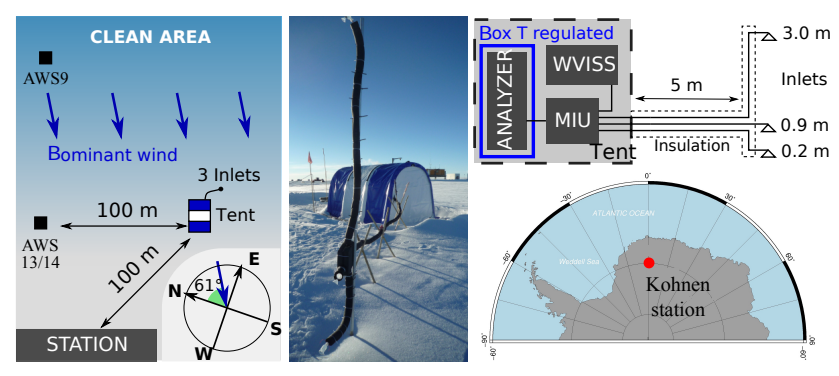

Figure 1. Location of Kohnen station in Antarctica (bottom right panel). Location of the two automatic weather stations (AWS, left panel) and location of the measurement tent connected to the three inlets (central panel). The right schematic presents the setup with the three inlets: the multiport (MIU), the Water Vapor Isotope Standard Source (WVISS) and the analyzer measuring the humidity mixing ratio $q$ and isotopes $\delta \mathrm{D}$ and $\delta^{18} \mathrm{O}$ in the vapor.

\subsection{Water vapor sampling system}

Measurements of water vapor isotopes in the near-surface atmosphere were performed from 17 December 2013 to 21 January 2014 using a Los Gatos Research Inc. analyzer (hereafter simply analyzer), type DTL-100. It continuously measured the humidity mixing ratio and the ratio of two stable water isotopes: $R_{18} \mathrm{O}=\left[{ }^{1} \mathrm{H}_{2}^{18} \mathrm{O}\right] /\left[{ }^{1} \mathrm{H}_{2}^{16} \mathrm{O}\right]$ and $R_{\mathrm{D}}=\left[{ }^{1} \mathrm{H}^{2} \mathrm{H}^{16} \mathrm{O}\right] /\left[{ }^{1} \mathrm{H}_{2}^{16} \mathrm{O}\right]$ (Baer et al., 2002). We will use throughout this paper the standard $\delta$ notation in \%o:

$\delta^{*}=\left(\frac{R_{*}}{R_{\mathrm{VSMOW}}}-1\right) \times 1000$,

where $\delta^{*}$ stands for $\delta \mathrm{D}$ or $\delta^{18} \mathrm{O}$, and $R_{\mathrm{VSMOW}}$ is the ratio of the Vienna Standard Mean Ocean Water. The instrumental temporal resolution is $2 \mathrm{~Hz}$, but we report measurements averaged over $11 \mathrm{~min}$ to increase the signal-to-noise ratio. The precision decreases with humidity, leading us to exclude all measurements performed below 500 ppmv.

The analyzer was calibrated using a stream of water vapor with known constant isotopic composition generated by the Water Vapor Isotope Standard Source (WVISS, Los Gatos Research Inc.). The WVISS allowed control over the amount of dilution of the vapor stream, resulting in a vapor stream of adjustable humidity level. A working standard was created at the beginning of the campaign by melting surface snow and subsequently stored in a sealed glass container. Samples were taken from the working standard every 2 weeks for later laboratory isotopic analysis to check for stability. No significant drift was observed. The working standard was calibrated against VSMOW, SLAP (Standard Light Antarctic Precipitation) and GISP (Greenland Ice Sheet Precipitation) standards at the Alfred Wegener Institute, Bremerhaven (hereafter AWI). Its isotopic composition was $\delta^{18} \mathrm{O}=-44.44 \pm 0.03 \%$ and $\delta \mathrm{D}=-345.5 \pm 0.1 \%$.

Figure 1 shows the location of the instrument compared to the main wind direction $\left(\sim 61^{\circ}\right.$ true north $)$ and its distance 
Table 1. Conversion slopes calculated from four VSMOW-SLAP calibrations with different standards. Data have been corrected with respect to humidity before calculating the slopes. Uncertainties represent $1 \mathrm{SE}$ (standard error) on the slopes.

\begin{tabular}{lccl}
\hline Day of calibration & $\begin{array}{c}\delta \mathrm{D} \text { (standards) vs. } \\
\delta \mathrm{D} \text { (measured) }\end{array}$ & $\begin{array}{c}\delta^{18} \mathrm{O} \text { (standards) vs. } \\
\delta^{18} \mathrm{O} \text { (measured) }\end{array}$ & Standards used \\
\hline 6 Dec 2013 & $1.45 \pm 0.02$ & $1.03 \pm 0.07$ & NZE, JASE, TD1 \\
15 Dec 2013 & $1.40 \pm 0.03$ & $0.93 \pm 0.05$ & DML, TD1, NZE, JASE \\
8 Jan 2014 & $1.38 \pm 0.01$ & $1.01 \pm 0.03$ & NZE, OC3, TALOS, NEEM \\
22 Jan 2014 & $1.47 \pm 0.01$ & $1.01 \pm 0.01$ & JASE, TD1, NZE, DML \\
\hline
\end{tabular}

to the base. The large clean area prevents any perturbations from local human activity. Measurements were performed at three different heights above the snow surface: $0.2,0.9$ and $3 \mathrm{~m}$. Insulated and heated copper tubes were used to suck in air to the analyzer following the setup of Steen-Larsen et al. (2013). An air filter and a snowfall protection were placed on each inlet to prevent sucking in snow crystals. The three copper tubes and the WVISS were connected to a device called the Multiport Input Unit (Los Gatos Research Inc.). This device was controlled by the analyzer to switch the valves and alternate between the inlet measurements and WVISS calibrations. Dry air was flushed through the system to check for leaks. The order of measurements during the campaign was the following: 12 min calibration followed by three cycles of 33 min, measuring $11 \mathrm{~min}$ at each inlet.

\subsection{Calibrations}

The calibration protocol follows Steen-Larsen et al. (2013). In short, one calibration is applied to the humidity mixing ratio and then three types of calibrations of the measured water vapor isotopes are performed: instrumental humidity-isotope response calibration, VSMOW-SLAP calibration and drift correction. More details about the calibration procedures are given in the Supplement.

The humidity mixing ratio is calibrated against the RH measured by the AWS9. This RH has been previously calibrated following the protocol of Anderson (1994), setting its maximum values equal to $100 \%$ of humidity. Then the RH is converted into the humidity mixing ratio $q$ using the surface pressure (Goff-Gratch equation with respect to ice) and the air temperature at $2 \mathrm{~m}$ height. We finally calculate a fit from AWS9 to analyzer $q$, with a second-order polynomial to get the function correcting the humidity mixing ratio measured during the campaign. This function is $f(q)=a+b \times q+c \times q^{2}$ with $a=80 \pm 80, b=0.59 \pm 0.14$ and $c=(0.23 \pm 0.06) \times 10^{-3}$. We have checked the linear relationship between $\delta \mathrm{D}$ and $q$ (shown in Table 5) with/without this calibration and conclude that the slope is not sensitive.

The instrumental humidity-isotope response calibration was obtained by varying the humidity level of the vapor stream produced by the WVISS while measuring the same standard. As the water vapor isotopic composition gener- ated by the WVISS is assumed to be constant, the observed variation of the water vapor isotopes can be attributed to the instrumental humidity response. Five humidity calibrations were performed during the campaign $(6,14,28$ December 2013 and 13, 28 January 2014). For each of them, we continuously generated a stream of water vapor from the "working standard water", using the WVISS during $\sim 20 \mathrm{~h}$. We forced the humidity to vary from $\sim 4000$ to $300 \mathrm{ppmv}$ by changing the amount of dilution. The humidity isotope response between calibration periods is assumed to vary linearly.

The VSMOW-SLAP calibration is carried out by measuring vapor from the WVISS generated when evaporating standards of known isotopic composition referenced against the VSMOW-SLAP scale. Four different water standards (NZE, NEEM, TALOS, OC3) with a known isotopic composition were brought to Kohnen station in 40 glass bottles of $10 \mathrm{cL}$. During a VSMOW-SLAP calibration, each standard was vaporized and measured during $15+15 \mathrm{~min}$ at two different humidity levels, which were used to check the accuracy of the humidity correction. This calibration lasted $\sim 6 \mathrm{~h}$ and has been reproduced four times on 6 and 15 December 2013 and 8 and 22 January 2014. Very small variations in the VSMOW-SLAP calibration slope were observed (see Table 1) and we have therefore simply calculated the mean value to obtain our conversion slopes : $\alpha_{\delta \mathrm{D}}=1.43 \pm 0.02$ and $\alpha_{\delta^{18} \mathrm{O}}=1.00 \pm 0.03$ (uncertainties are the standard error of the mean value).

Finally the measured isotopic value is corrected for the drift by measuring vapor generated by the WVISS when evaporating the prepared working standard. This measurement is performed every $111 \mathrm{~min}$ during $12 \mathrm{~min}$ and linear interpolation is assumed between each drift-correction measurement.

\subsection{Precision and accuracy of measurements}

We use the following notation to describe the error propagation, with $\delta^{*}$ standing either for $\delta \mathrm{D}$ or $\delta^{18} \mathrm{O}$.

1. The raw isotopic composition (direct output from the analyzer without any corrections) averaged over $11 \mathrm{~min}$ is $\delta_{\text {raw }}^{*} \pm \mathrm{d} \delta_{\text {raw }}^{*}$, where $\mathrm{d} \delta_{\text {raw }}^{*}$ is the standard error associated with the mean value. 
2. The humidity isotope response associated with $\delta_{\text {raw }}^{*}$ is $\Gamma_{*} \pm \mathrm{d} \Gamma_{*}$, where $\mathrm{d} \Gamma_{*}$ is the uncertainty associated with the humidity correction. $\mathrm{d} \Gamma_{*}$ is, for a given $q$ and a given time, the absolute difference between two humidity isotope responses from two consecutive humidity calibrations. $\mathrm{d} \Gamma_{*}$ is therefore taken as the maximum possible error on the humidity correction.

3. The slope conversion to the VSMOW-SLAP international scale is $\alpha_{*} \pm \mathrm{d} \alpha_{*}$, where $\mathrm{d} \alpha_{*}$ is the uncertainty associated with the slope. $\mathrm{d} \alpha_{*}$ is the standard error of the mean value of the slopes from the four different VSMOW calibrations.

4. The drift correction is $\mu_{*} \pm \mathrm{d} \mu_{*}$, where $\mathrm{d} \mu_{*}$ is the uncertainty associated with the drift correction. $\mathrm{d} \mu_{*}$ has been estimated at the end of the campaign by performing an extra $24 \mathrm{~h}$ calibration with a stable humidity.

5. The corrected isotopic composition is $\delta_{\text {corr }}^{*} \pm \mathrm{d} \delta_{\text {corr }}^{*}$, where $\mathrm{d} \delta_{\text {corr }}^{*}$ is the final uncertainty containing both the precision and the accuracy on the corrected measurements.

We apply the three corrections (humidity isotope response, conversion to the VSMOW-SLAP scale, drift removal) to calculate the corrected isotopic composition at a given time:

$\delta_{\text {corr }}^{*}=\alpha_{*} \times\left(\delta_{\text {raw }}^{*}-\Gamma_{*}\right)-\mu_{*}$.

We obtain a final uncertainty on the corrected isotopic composition by applying an error propagation calculation, assuming no correlation between the three corrections:

$\left(\delta_{\text {corr }}^{*}\right)^{2}=P_{*}^{2}+A_{*}^{2}$,

with

$P_{*}^{2}=\alpha_{*}^{2} \times\left(\left(\mathrm{d} \delta_{\text {raw }}^{*}\right)^{2}+\left(\mathrm{d} \Gamma_{*}\right)^{2}\right)+\left(\mathrm{d} \mu_{*}\right)^{2}$

$A_{*}^{2}=\left(\mathrm{d} \alpha_{*}\right)^{2} \times\left(\delta_{\text {raw }}^{*}-\Gamma_{*}\right)^{2}$.

The parameter $A^{*}$ stands for the accuracy of the measurements and $P^{*}$ stands for the precision of the measurements. We have attributed the part $A^{*}$ to the accuracy because the uncertainty on the VSMOW correction will affect the mean value of the data over the campaign. Each correction depends on the time of the measurement (the drift varies through time, as does the humidity isotope response) and Table 2 summarizes the different orders of magnitude of the parameters with the estimated precision / accuracy.

We estimate a precision of the measurements of $3.0 \%$ for $\delta \mathrm{D}$ and of $0.9 \%$ for $\delta^{18} \mathrm{O}$. We estimate an accuracy of the measurements of $11 \%$ for $\delta \mathrm{D}$ and of $2.5 \%$ for $\delta^{18} \mathrm{O}$. When focusing on the mean diurnal cycle, we will get a higher precision by calculating hourly averages over 18 days (Sect. 4.2).
Table 2. Order of magnitude of the parameters involved in the error propagation calculation for $\delta \mathrm{D}$ and $\delta^{18} \mathrm{O}$. "avg", "min" and "max" are the mean, the minimum and the maximum value of the parameter over the campaign. $A$ stands for accuracy and $P$ for precision. Every parameter is in \%o except $\alpha_{*}$ and $\mathrm{d} \alpha_{*}$, which are dimensionless.

\begin{tabular}{llllllll}
\hline & \multicolumn{3}{c}{ Related to $\delta \mathrm{D}$} & & \multicolumn{3}{c}{ Related to $\delta^{18} \mathrm{O}$} \\
\cline { 2 - 3 } \cline { 7 - 8 } & Avg & Min & Max & & Avg & Min & Max \\
\hline$\delta_{\text {raw }}^{*}$ & -552 & -586 & -519 & & -84.3 & -94.6 & -77.7 \\
$\mathrm{~d} \delta_{\text {raw }}^{*}$ & 0.3 & 0.1 & 0.9 & & 0.1 & 0.05 & 0.4 \\
\hline$\left|\Gamma_{*}\right|$ & 4 & 0 & 9 & & 1.7 & 0 & 4 \\
$\mathrm{~d} \Gamma_{*}$ & 2 & 0.1 & 5 & & 0.4 & 0 & 2 \\
\hline$\alpha_{*}$ & 1.43 & 1.38 & 1.47 & & 1.00 & 0.93 & 1.03 \\
$\mathrm{~d} \alpha_{*}$ & 0.02 & - & - & 0.03 & - & - \\
\hline $\mathrm{d} \mu_{*}$ & 1.4 & - & - & & 0.7 & - & - \\
\hline$A_{*}$ & 11 & 10 & 12 & 2.5 & 2.2 & 2.9 \\
$P_{*}$ & 3.0 & 1.4 & 7.3 & 0.9 & 0.7 & 2.0 \\
\hline
\end{tabular}

\subsection{Surface snow sampling and analysis}

In order to detect any snow accumulation or erosion (due to snowfall events or wind drift), 100 thin wood sticks were distributed every meter along a $100 \mathrm{~m}$ transect in a clean area and daily measured with a folding ruler. No accumulation or erosion was detected within a precision of $1 \mathrm{~mm}$.

The Snow Surface Diurnal Cycle (SSDC) experiment was devoted to the detection of a diurnal cycle in the isotopic composition of surface snow. Keeping in mind the possible variability in the isotopic composition of the snow surface, three different areas with consistent surface snow texture were selected, based on visual observation (the border of the snow patch was visible) and subjective assessment of the hardness. The snow sampling protocol is based on the assumption that the isotopic composition of a snow patch at a given time is homogeneous. Patch 1 was made of hard ice, patch 2 of compact snow and patch 3 was composed of soft snow. Five adjacent samples for each patch were sampled every hour ( 15 samples per hour) during a $35 \mathrm{~h}$ period, from 8 to 10 January 2014 (as it is shown in Fig. 2 indicated by the SSDC label). The sample depth is estimated to be between 2 and $5 \mathrm{~mm}$; the tool used was a cake shovel. Samples were scraped into a plastic bag, which was sealed and shipped for subsequent isotope measurements at the AWI, using two water isotope analyzers Picarro: type L1102-i and L2120-i. The protocol followed Geldern and Barth (2012).

\subsection{Atmospheric simulations}

ECHAM5-wiso (Werner et al., 2011) is the isotopic version of the atmospheric general circulation model ECHAM5 (Roeckner et al., 2003). Simulations from ECHAM5-wiso are implicitly nudged to the European Center for Medium- 


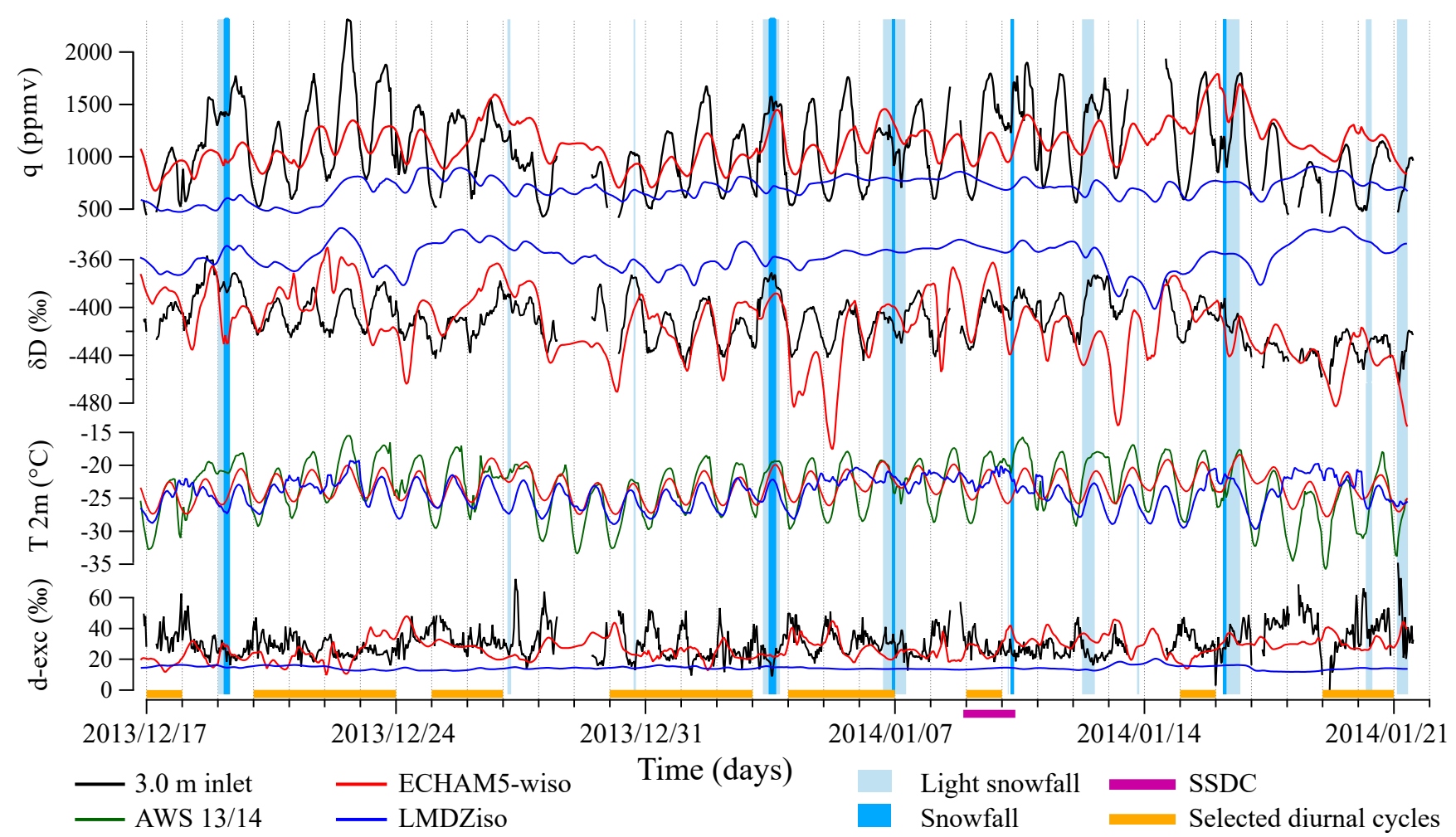

Figure 2. Hourly observed (black and green) and simulated (red and blue) humidity mixing ratio $(q)$, deuterium $(\delta \mathrm{D})$, air temperature at $2 \mathrm{~m}$ ( $T 2 \mathrm{~m}$, measured with the AWS from the 2013/2014 season) and d-excess (d-exc) at Kohnen station 2013/2014. Hourly observed precipitation events are labeled light snowfall and snowfall (light and dark cyan). The Snow Surface Diurnal Cycle (SSDC) experiment period is indicated in purple, and the 18 selected days for the diurnal cycles study are in orange.

Range Weather Forecasts (ECMWF) ERA-interim reanalyses data (Berrisford et al., 2011) using 6-hourly pressure, temperature, divergence and vorticity fields (Rast et al., 2013). Sea surface temperatures and sea ice coverage are derived from the ERA-interim dataset too. For our purpose this model has been run with a high vertical and horizontal T106L 31 resolution (31 levels, $1.1^{\circ}$ in longitude $\times 1.1^{\circ}$ in latitude). The lowest model level (about $60 \mathrm{~m}$ above the surface) has been selected followed by a bilinear interpolation of nearby model grid points to the location of Kohnen base $\left(75^{\circ} 00^{\prime} \mathrm{S}, 0^{\circ} 04^{\prime} \mathrm{E}\right)$. The simulation was started in 1979 and any potential model spinup bias, for example, caused by the initialization of the atmosphere in terms of humidity and its isotopic composition, can be safely neglected for our study period.

LMDZ5Aiso (hereafter LMDZiso) is the isotopic version of LMDZ5A, part of the atmospheric general circulation model IPSL-CM5A used in the Coupled Model Intercomparison Project (CMIP5) (Risi et al., 2010). For our purpose this model has been run with a vertical resolution of 39 levels and with a stretched grid designed to refine the horizontal resolution in Antarctica: the resolution is of $0.30^{\circ}$ latitude $\times 2.5^{\circ}$ longitude in Antarctica, yielding a resolution of about $33 \mathrm{~km}$ in latitude $\times 72 \mathrm{~km}$ in longitude around Kohnen. Simulations are constrained by sea surface temperature (SST) data from the National Centers for Environmental Prediction (NCEP) and nudged to the 6-hourly ECMWF analyses using only the wind fields. There could theoretically be some inconsistencies between the winds and the SSTs from different reanalyses datasets, but the impact should be very small due to the overall consistency between the two reanalyses datasets and due to the strong nudging of the winds, preventing any drift. Because such high-resolution simulation is costly, the simulation has been started in January 2013 but inspection of simulated time series show that the spinup in sufficient.

Three selected outputs from both models are calculated at a specific height, $10 \mathrm{~m}$ for the wind speed and wind direction, and at $2 \mathrm{~m}$ for temperature. However, the reader should notice that simulated parameters such as humidity or water vapor isotopes are extracted from the first vertical model level (which represents a height of $60 \mathrm{~m}$ above ground) whereas the in situ observations are measured close to the surface. Furthermore, at Kohnen Station, the $2 \mathrm{~m}$ temperature in ECHAM5-wiso is calculated from the surface energy balance equation, assuming a constant surface albedo of 0.8. This might also lead to further differences between simulation results and observations. 


\section{Results and discussion}

Section 4.1 will be devoted to the study of the day-to-day variability over the study period of both air observations at $3 \mathrm{~m}$ and simulations from ECHAM5-wiso and LMDZiso. Section 4.2 will focus on the diurnal scale, showing subtle differences between the 0.2 and $3 \mathrm{~m}$ inlets in the mean value of 18 selected days (labeled as horizontal orange bars in Fig. 2) and comparing it with AGCM outputs. Finally, Section 4.3 will study the diurnal cycle in the snow surface, by comparing the results from the snow surface samples collected during the SSDC experiment (labeled as the horizontal purple bar in Fig. 2) with isotopic simulations from a snow surface-air model running as a closed system.

\subsection{Observed and simulated day-to-day variability}

In order to estimate the magnitude of the day-to-day variability, days with data gaps larger than $8 \mathrm{~h}$ have been removed from the dataset (on 16, 28 and 29 December and 13, 14, 17, 18 and 21 January) and then 29 daily mean values of $q, T$, dexcess and $\delta \mathrm{D}$ have been calculated for the observations and the model outputs. Average and standard deviation of these parameters over the 29 mean values are detailed in Table 3 .

The signal from the temperature, humidity mixing ratio and water vapor isotopes measurements is stable, with a much lower variability on the day-to-day scale compared to the diurnal scale (Fig. 2). The mean humidity mixing ratio measured at $3 \mathrm{~m}$ over the study period is $1100 \mathrm{ppmv}$ (Table 3), with a maximum of $2200 \mathrm{ppmv}$ on 22 December at 15:00 UTC. It coincides with the highest temperature, reaching $-15.5^{\circ} \mathrm{C}$ at $17: 00 \mathrm{UTC}$ on the same day. By contrast, the driest and coldest conditions are encountered on 19 January, at 02:00 UTC, and is estimated at $150 \mathrm{ppmv}$ for the humidity mixing ratio (therefore below the limit of confidence of our instrument, $500 \mathrm{ppmv}$ ) and measured at $-35.8^{\circ} \mathrm{C}$ for the air temperature. Deuterium mean value at $3 \mathrm{~m}$ is $-410 \%$, with a range of variation from $-360 \%$ (on 18 December at 17:00 UTC) to $-470 \%$ (on 21 January at 03:00 UTC).

We now compare the model performances for these daily mean values. ECHAM5-wiso correctly simulates these mean conditions for the temperature, humidity mixing ratio and deuterium while LMDZiso produces a mean humidity mixing ratio $40 \%$ lower than observed and shows a positive offset of $56 \%$ in deuterium (Table 3). We notice that the mean radiative input (in longwave and shortwave) measured at the surface by the AWS 9 is $583 \mathrm{~W} \mathrm{~m}^{-2}$, compared to only $552 \mathrm{~W} \mathrm{~m}^{-2}$ for LMDZiso. An incorrect simulation of the cloud cover (and subsequently the precipitation) is likely related to this offset in LMDZiso. The surface energy balance determines the mean surface temperature $\left(-27^{\circ} \mathrm{C}\right.$ for LMDZiso compared to $-24^{\circ} \mathrm{C}$ for ECHAM5-wiso), which itself impacts the sublimation rate and the $2 \mathrm{~m}$ air temperature via sensible and latent heat exchanges with the lower atmosphere. The radiative offset present in LMDZiso could
Table 3. Mean values over 29 daily averages from the measurement period (days with more than $8 \mathrm{~h}$ of data gap have been removed). Uncertainties represent $\pm 1 \mathrm{SD}$ (standard deviation) of the mean value. ECHAM stands for ECHAM5-wiso.

\begin{tabular}{lrlll}
\hline & $q$ (ppmv) & $\delta \mathrm{D}(\%)$ & $\mathrm{d}$-exc $(\% o)$ & $T 2 \mathrm{~m}\left({ }^{\circ} \mathrm{C}\right)$ \\
\hline AWS 13/14 & - & - & - & $-23 \pm 4$ \\
$0.2 \mathrm{~m}$ inlet & $1000 \pm 200$ & $-413 \pm 9$ & $33 \pm 11$ & - \\
$3.0 \mathrm{~m}$ inlet & $1100 \pm 200$ & $-409 \pm 9$ & $30 \pm 9$ & - \\
ECHAM & $1120 \pm 110$ & $-411 \pm 15$ & $26 \pm 5$ & $-23.1 \pm 1.7$ \\
LMDZiso & $700 \pm 100$ & $-355 \pm 10$ & $15 \pm 1$ & $-24.0 \pm 1.4$ \\
\hline
\end{tabular}

explain the low simulated values of temperature and humidity mixing ratio.

Observations depict a day-to-day variability of $200 \mathrm{ppmv}$ for the humidity mixing ratio, $9 \%$ for deuterium and $4{ }^{\circ} \mathrm{C}$ for temperature (Table 3). Both models underestimate the variability of temperature and humidity mixing ratio. One explanation concerning temperature could be that both models fail to capture the very cold events observed on $30 \mathrm{De}-$ cember and 19 January. They also underestimate the variations of temperature and humidity mixing ratio observed over several days of relatively clear sky from 20 to 24 December, with an amplitude in $T(q)$ of $0.9^{\circ} \mathrm{C}(25 \mathrm{ppmv})$ for ECHAM5-wiso against $4.2^{\circ} \mathrm{C}$ (490 ppmv) for the observations. The deuterium has a higher day-to-day variability in ECHAM5-wiso because of the simulation of high-depletion events which strongly deviate from the observations (Fig. 2). For example, on 5 January at 06:00 UTC the simulated value is as low as $-520 \%$ against $-430 \%$ for the top inlet. These depletion events do not correspond to any parallel signal in the simulated meteorological data (cloud cover, wind speed, temperature or humidity mixing ratio) and further analyses will be necessary to understand these artifacts.

We finally focus on deuterium excess. The observed mean value at $3 \mathrm{~m}$ is $\sim 30 \%$, with a strong relative variability of 9\%o (Table 3). ECHAM5-wiso simulates deuterium excess values close to the observations within these uncertainties, while LMDZiso shows a low signal of $15 \%$ with almost no variability. Some of the air masses simulated by ECHAM5wiso have even a correct deuterium excess variability despite the fact that the model is not able to simulate correctly the magnitude of the $\delta \mathrm{D}$ depletion during these days, as reported above (e.g., on 5 and 13 January). This might be explained by a stronger dependency of deuterium excess variability to climate conditions during evaporation processes in the vapor source regions, while the $\delta \mathrm{D}$ signal is understood to be more directly controlled by climate conditions near or at Kohnen station.

Table 5 presents a synthesis of the linear relationships between the deuterium and different parameters (temperature, humidity mixing ratio, $\delta^{18} \mathrm{O}$ and d-excess) for the observed and simulated values on two different timescales (day-to-day and diurnal). The left column corresponds to the 29 daily 
Table 4. Peak-to-peak amplitude of the 18 selected diurnal cycles. Uncertainties represent \pm 1 SD (average of the 24 SD of the hourly mean values). ECHAM stands for ECHAM5-wiso.

\begin{tabular}{lrrrr}
\hline & $\Delta q(\mathrm{ppmv})$ & $\Delta \delta \mathrm{D}(\% \circ)$ & $\Delta \mathrm{d}-\operatorname{exc}(\% \circ)$ & $\Delta T 2 \mathrm{~m}\left({ }^{\circ} \mathrm{C}\right)$ \\
\hline AWS 13/14 & - & - & - & $10.1 \pm 1.0$ \\
$0.2 \mathrm{~m}$ inlet & $1010 \pm 130$ & $40 \pm 7$ & $21 \pm 7$ & - \\
$3.0 \mathrm{~m}$ inlet & $930 \pm 120$ & $36 \pm 6$ & $15 \pm 6$ & - \\
ECHAM & $280 \pm 60$ & $32 \pm 15$ & $7 \pm 4$ & $4.9 \pm 0.4$ \\
LMDZiso & $160 \pm 100$ & $7 \pm 4$ & $0.6 \pm 0.4$ & $3.0 \pm 1.3$ \\
\hline
\end{tabular}

mean values we have calculated before. Only $\delta \mathrm{D}$ vs $\delta^{18} \mathrm{O}$ presents a strong linear relationship on this timescale, because the day-to-day variability of other parameters (mixing ratio, temperature) is much weaker than their diurnal variations (Sect. 4.2). The strong linear relationship on the diurnal scale highlights the importance of the local processes, which will be investigated through a box model in Sect. 4.3.

\subsection{Observed and simulated diurnal cycles}

We have chosen 18 days showing a strong diurnal variation in the humidity mixing ratio and the isotopes for the purpose of stacking them: for each of these days and for each parameter, the daily mean value has been subtracted to obtain the anomalies. Then from these anomalies we have calculated for each hour (from 01:00 to 24:00 UTC) the average of the 18 values and its associated standard deviation. For visualization purposes, we have calculated the average of the $24 \mathrm{SD}$ (standard deviations), called hereafter MSD for mean SD (each error bar in Fig. 3 represents \pm 1 MSD). The MSD associated with the wind direction has been obtained differently, because this parameter is calculated from the $U$ and $V$ wind components. Knowing the standard deviation $\sigma_{U}$ and $\sigma_{V}$ associated with the $U$ and $V$ stacks, a Monte Carlo simulation has been performed to calculate for each run a different wind direction from random values of $U \pm 2 \sigma_{U}$ and $V \pm 2 \sigma_{V}$. The MSD associated with the wind direction is the average of the maximum range of values obtained from the Monte Carlo simulation $(n=10000)$. Also, the saturated mixing ratio has been shifted to preserve the difference $\left(q_{\text {sat }}-q\right)$ and allow a meaningful comparison with the humidity mixing ratio anomalies. The average temperature (average humidity mixing ratio at $3 \mathrm{~m}$ ) over the 18 selected days is $-23.6 \pm 1.9^{\circ} \mathrm{C}$ $(1130 \pm 170 \mathrm{ppmv})$, which is close to the mean values over the campaign $\left(-23^{\circ} \mathrm{C}, 1100 \mathrm{ppmv}\right)$ and indicates that these days constitute a representative sample of the campaign. We will also frequently refer to the measurements performed by Van As et al. (2005) at Kohnen from 8 January to 9 February 2002 in temperature and specific humidity at two different heights: surface and $1 \mathrm{~m}$.

The mean wind speed is $4.4 \pm 0.1 \mathrm{~m} \mathrm{~s}^{-1}$ over the $18 \mathrm{se}-$ lected days, with a diurnal variation from $5.8 \mathrm{~m} \mathrm{~s}^{-1}$ (12:00 UTC) to $2.4 \mathrm{~m} \mathrm{~s}^{-1}$ (20:00 UTC). The mean wind direction over the 18 selected days is $46 \pm 50^{\circ}$. These mea-
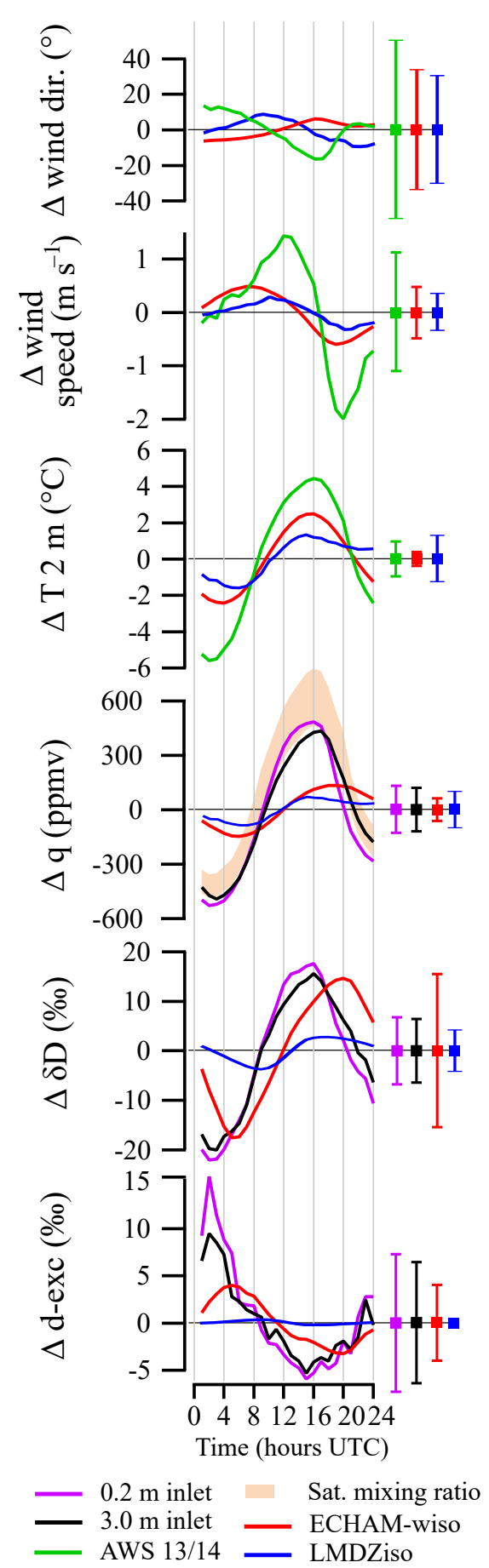

Figure 3. Stack of 18 diurnal cycles of the wind direction and wind speed (simulated at $10 \mathrm{~m}$ for the models, measured at $2 \mathrm{~m}$ for the observations), air temperature at $2 \mathrm{~m}$ (measured with the AWS from the 2013/2014 season), humidity mixing ratio $q$, saturated mixing ratio range calculated with the surface pressure and temperature at $2 \mathrm{~m} \pm 1{ }^{\circ} \mathrm{C}$, deuterium $\delta \mathrm{D}$ and d-excess. Error bars represent $\pm 1 \mathrm{MSD}$ (mean standard deviation): average of the $24 \mathrm{SD}$ associated with the hourly mean values. 
Table 5. Slopes and determination coefficients calculated from two datasets: (1) the 29 daily mean values from the campaign and (2) the 24 hourly mean values from the stack composed of 18 selected diurnal cycles. Uncertainties represent $1 \mathrm{SE}$ on the slopes.

\begin{tabular}{|c|c|c|}
\hline & $\begin{array}{l}\text { Daily mean } \\
\text { values }(n=29)\end{array}$ & $\begin{array}{l}\text { Hourly mean } \\
\text { values }(n=24)\end{array}$ \\
\hline \multicolumn{3}{|c|}{$\delta \mathrm{D}(\% \circ)$ vs. $q(\mathrm{ppmv})$} \\
\hline $3.0 \mathrm{~m}$ inlet & $\begin{array}{l}\alpha=0.04 \pm 0.01 \\
r^{2}=0.31\end{array}$ & $\begin{array}{l}\alpha=0.037 \pm 0.001 \\
r^{2}=0.98\end{array}$ \\
\hline ECHAM5-wiso & $\begin{array}{l}\alpha=0.03 \pm 0.02 \\
r^{2}=0.05\end{array}$ & $\begin{array}{l}\alpha=0.071 \pm 0.008 \\
r^{2}=0.76\end{array}$ \\
\hline LMDZiso & $\begin{array}{l}\alpha=0.06 \pm 0.01 \\
r^{2}=0.37\end{array}$ & $\begin{array}{l}\alpha=0.034 \pm 0.005 \\
r^{2}=0.69\end{array}$ \\
\hline \multicolumn{3}{|c|}{$\delta \mathrm{D}(\% \circ)$ vs. $T 2 \mathrm{~m}\left({ }^{\circ} \mathrm{C}\right)$} \\
\hline $3.0 \mathrm{~m}$ inlet & $\begin{array}{l}\alpha=3.1 \pm 1.1 \\
r^{2}=0.24\end{array}$ & $\begin{array}{l}\alpha=3.37 \pm 0.11 \\
r^{2}=0.98\end{array}$ \\
\hline ECHAM5-wiso & $\begin{array}{l}\alpha=6 \pm 4 \\
r^{2}=0.10\end{array}$ & $\begin{array}{l}\alpha=4.7 \pm 0.9 \\
r^{2}=0.54\end{array}$ \\
\hline LMDZiso & $\begin{array}{l}\alpha=3.9 \pm 1.1 \\
r^{2}=0.33\end{array}$ & $\begin{array}{l}\alpha=1.5 \pm 0.3 \\
r^{2}=0.49\end{array}$ \\
\hline \multicolumn{3}{|c|}{$\delta \mathrm{D}(\% o)$ vs. $\delta^{18} \mathrm{O}\left(^{\circ}\right)$} \\
\hline $3.0 \mathrm{~m}$ inlet & $\begin{array}{l}\alpha=6.2 \pm 0.3 \\
r^{2}=0.94\end{array}$ & $\begin{array}{l}\alpha=5.99 \pm 0.12 \\
r^{2}=0.99\end{array}$ \\
\hline ECHAM5-wiso & $\begin{array}{l}\alpha=6.64 \pm 0.19 \\
r^{2}=0.98\end{array}$ & $\begin{array}{l}\alpha=6.57 \pm 0.04 \\
r^{2}=0.99\end{array}$ \\
\hline LMDZiso & $\begin{array}{l}\alpha=7.5 \pm 0.1 \\
r^{2}=0.99\end{array}$ & $\begin{array}{l}\alpha=7.41 \pm 0.02 \\
r^{2}=0.99\end{array}$ \\
\hline \multicolumn{3}{|c|}{ d-excess $(\% o)$ vs. $\delta \mathrm{D}(\% \circ)$} \\
\hline $3.0 \mathrm{~m}$ inlet & $\begin{array}{l}\alpha=-0.21 \pm 0.06 \\
r^{2}=0.32\end{array}$ & $\begin{array}{l}\alpha=-0.32 \pm 0.03 \\
r^{2}=0.86\end{array}$ \\
\hline ECHAM5-wiso & $\begin{array}{l}\alpha=-0.18 \pm 0.03 \\
r^{2}=0.50\end{array}$ & $\begin{array}{l}\alpha=-0.22 \pm 0.01 \\
r^{2}=0.98\end{array}$ \\
\hline LMDZiso & $\begin{array}{l}\alpha=-0.06 \pm 0.02 \\
r^{2}=0.34\end{array}$ & $\begin{array}{l}\alpha=-0.08 \pm 0.01 \\
r^{2}=0.97\end{array}$ \\
\hline
\end{tabular}

surements are therefore consistent with the presence of katabatic winds toward the slope direction of the terrain $\left(61^{\circ}\right)$. Both models fail to simulate the pattern of katabatic winds. The mean wind direction is $32 \pm 27^{\circ}$ for ECHAM5-wiso and $21 \pm 24^{\circ}$ for LMDZiso, and their mean wind velocity at $10 \mathrm{~m}$ is only $3.1 \mathrm{~m} \mathrm{~s}^{-1}$ for ECHAM5-wiso and $1.9 \mathrm{~m} \mathrm{~s}^{-1}$ for LMDZiso. They also show a low diurnal variability, whereas Van As et al. (2005) observed at the same height variations higher than $2 \mathrm{~m} \mathrm{~s}^{-1}$ over $24 \mathrm{~h}$. The underestimation might be due to the horizontal resolution, which is too coarse to represent properly the katabatic winds, especially in LMDZiso.
We focus on the peak-to-peak amplitude of the diurnal cycles, summarized in Table 4. The diurnal amplitude of observed surface air temperature at $2 \mathrm{~m}$ is $10.0^{\circ} \mathrm{C}$ (within a MSD of $1.0^{\circ} \mathrm{C}$ ). Van As et al. (2005) found an amplitude of $\sim 11^{\circ} \mathrm{C}$ at $1 \mathrm{~m}$, which is consistent with our observations. The diurnal amplitude observed at $3 \mathrm{~m}$ is $930 \mathrm{ppmv}$ for $q$ and $36 \%$ for $\delta \mathrm{D}$. The diurnal variation in the water vapor isotopes and temperature is similar at NEEM in Greenland $\left(\sim 35 \%\right.$ o for $\delta \mathrm{D}$ and $\sim 10^{\circ} \mathrm{C}$ for $T$ ), despite much larger diurnal variations in the humidity mixing ratio with a peak-topeak amplitude of $\sim 2300 \mathrm{ppmv}$ (Steen-Larsen et al., 2013).

Both models underestimate the amplitude of the diurnal cycles in the air temperature at $2 \mathrm{~m}$ by more than $50 \%$. The surface temperature simulated by both AGCMs has a peakto-peak amplitude of $7{ }^{\circ} \mathrm{C}$, compared to $\sim 14^{\circ} \mathrm{C}$ for the measurements of Van As et al. (2005). Variations of the surface temperature at Kohnen are supposed to be driven on the first order by the radiative budget. We have therefore compared the radiative budget of the AWS 9, ECHAM5-wiso and LMDZiso. Both models show good agreement with the observations for the net shortwave budget at the surface. However, the longwave radiative components are more difficult to simulate. Downward longwave emissions are related to the cloud cover (greenhouse effect) and snowfalls, whereas upward longwave emissions are related to the surface temperature and emissivity of the surface. Both models show difficulties simulating a proper cloud cover and snowfall events, and the variation in their surface temperature is $50 \%$ lower than observed. That explains the disagreement between the observations and the AGCMs with respect to the longwave radiative budget, leading to a wrong simulation of the surface temperature. Both AGCMs simulate a lower amplitude for the humidity mixing ratio likely due to the low variation in their surface temperature, but interestingly ECHAM5-wiso manages to simulate the right magnitude of the diurnal deuterium variability $(32 \%)$, associated with a large MSD of $15 \%$ o (Table 4). The model-data comparison is hampered by the fact that the simulated humidity mixing ratio is only available from the first grid level of the AGCMs and is therefore an average value over the first $\sim 60 \mathrm{~m}$. The height and stability of the boundary layer are particularly difficult to simulate over ice and have a certain impact on the presence or absence of diurnal cycles (Holtslag et al., 2013). A proper understanding of the simulation of the boundary layer by the AGCMs would require relevant output parameters such as the boundary layer depth or stability classes, which have not been implemented yet. Further analyses will therefore be necessary to understand the different behavior of LMDZiso and ECHAM5-wiso.

We now compare the amplitudes at 0.2 and $3 \mathrm{~m}$ for the humidity mixing ratio and the $\delta \mathrm{D}$. The absolute difference between the amplitudes measured from the bottom and the top inlets is 80 ppmv for $q(20 \%$ of $\Delta q)$ and $4 \%$ o for $\delta \mathrm{D}$. Within a MSD of $\sim 125$ ppmv for $q$ and $\sim 6 \%$ for $\delta \mathrm{D}$, we cannot 
conclude that the amplitude is decreasing significantly with height.

In the observations, diurnal air temperature variations occur in phase with $\delta \mathrm{D}$ and humidity mixing ratio measured at $0.2 \mathrm{~m}$, with minima at 02:00 UTC and maxima at 16:00 UTC. While $\delta \mathrm{D}$ observations from 0.2 and $3 \mathrm{~m}$ seem synchronous within uncertainties, the top inlet presents a clear lag of $1 \mathrm{~h}$ behind the bottom inlet with respect to the measured humidity mixing ratio. The same delay was observed by Van As et al. (2005) from the surface to $1 \mathrm{~m}$ in both temperature and humidity mixing ratio. Their observations showed minima and maxima for $T$ and $q$ at $\sim 03: 00$ and $\sim 16: 00$ UTC at $1 \mathrm{~m}$ against $\sim 02: 00$ and $\sim 15: 00 \mathrm{UTC}$ at the surface. The diurnal observations from NEEM (Steen-Larsen et al., 2013) were also in phase with $T, q$ and $\delta \mathrm{D}$ at $\sim 1 \mathrm{~m}$; however, humidity mixing ratio and $\delta \mathrm{D}$ measured on a much higher tower $(\sim 13 \mathrm{~m})$ showed a delay of only $\sim 1 \mathrm{~h}$ with the observations at $1 \mathrm{~m}$. In the simulations from ECHAM5-wiso and LMDZiso, temperature diurnal variations occur approximately synchronous with the observations at Kohnen. However, simulated humidity mixing ratio and deuterium variations are delayed by $\sim 3 \mathrm{~h}$ compared to the observations from the 0.2 and $3 \mathrm{~m}$ inlets. This could be explained by the fact that the temperature at $2 \mathrm{~m}$ is driven by the surface radiative budget while the timing of changes in humidity/isotopes may reflect boundary layer dynamics which are less accurately simulated.

The d-excess values also depict a diurnal cycle anticorrelated to $\delta \mathrm{D}$, with an amplitude for the $0.2 \mathrm{~m}$ inlet $(3 \mathrm{~m}$ inlet) of $21 \%$ o $(15 \%$ ) but associated with a large MSD of $7 \%$ (6\%o). This anti-correlation is expected from the dexcess linear definition: at very low temperature, d-excess is influenced by distillation and increases as $\delta \mathrm{D}$ decreases. ECHAM5-wiso underestimates the diurnal amplitude variability and LMDZiso fails to simulate any diurnal variability in the d-excess.

As previously reported for daily mean values (Table 5, Sect. 4.1), close linear relationships are observed between $q, \delta \mathrm{D}$ and d-excess for hourly mean values, highlighting the importance of local fluxes. These relationships are better simulated for the diurnal cycle, but ECHAM5-wiso tends to overestimate the associated slopes compared to the observations. LMDZiso strongly overestimates the slope of $\delta \mathrm{D}$ $\delta^{18} \mathrm{O}$. ECHAM5-wiso is able to capture the diurnal anticorrelation of d-excess and $\delta \mathrm{D}$. The slope calculated on the hourly scale for $\delta \mathrm{D}$ vs. $\delta^{18} \mathrm{O}$ has a value of $5.99 \pm 0.12$, whereas Steen-Larsen et al. (2013) calculated at NEEM a slope of $6.47 \pm 0.07$, in a warmer and more humid air ( $\sim 3000$ ppmv against $\sim 1200 \mathrm{ppmv}$ at Kohnen).

\subsection{Air-snow exchanges}

In order to document the isotopic exchange between the surface snow and the overlying water vapor, snow surface samples were collected from three snow patches, with five jux-
Table 6. Isotopic mean values over the SSDC period. The standard error on the mean value is below $1 \%$ for $\delta \mathrm{D}$ and below $0.1 \%$ for $\delta^{18} \mathrm{O}$.

\begin{tabular}{lcc}
\hline & $\delta \mathrm{D}(\%)$ & $\delta^{18} \mathrm{O}(\%)$ \\
\hline Air observations & -407 & -54.4 \\
Soft patch & -296 & -37.4 \\
Medium patch & -301 & -37.0 \\
Hard patch & -316 & -38.8 \\
\hline
\end{tabular}

taposed replicas per patch and per hour (protocol described in Sect. 3.5). In the isotopic composition of the snow surface presented in Fig. 4, each hourly data point is the average of the five snow samples, with a standard deviation of $0.03 \%$ o for $\delta^{18} \mathrm{O}$ and $0.2 \%$ for $\delta \mathrm{D}$. We identify a clear diurnal cycle in the snow with a significant peak-to-peak amplitude of $\sim 3 \%$ o for $\delta \mathrm{D}$ and $\sim 0.4 \%$ o for $\delta^{18} \mathrm{O}$, in phase with the diurnal cycle in the air (Fig. 4). The mean deuterium value of the snow patches varies from -296 to $-316 \%$, showing that the texture of the snow patch and its isotopic composition could be related (Table 6). This observation confirms the spatial variability previously observed at Vostok in the isotopic composition of the snow surface $(10 \mathrm{~cm}$ depth), with variations up to $30 \%$ in $\delta \mathrm{D}$ over $100 \mathrm{~m}$ horizontally (Ekaykin et al., 2002). Both AGCMs manage to simulate a similar isotopic composition for the snow surface, with on average a deuterium value of $-330 \%$ or ECHAM5-wiso and $-299 \%$ for LMDZiso. As expected, the isotopic composition of the snow surface simulated by the AGCMs depends on the snowfall events only, with a variation in $\delta \mathrm{D}$ of $6 \%$ for ECHAM5-wiso over the study period (no variation is simulated by LMDZiso). Unfortunately, the cooling phase during the night of 9 January 2014 is restricted compared to the usual strong decrease in temperature or humidity shown in Fig. 3 because the presence of a cloud cover. This meteorological event is likely to have impacted $T, q$ and $\delta \mathrm{D}$ during the cooling phase.

We notice that the vapor is close to or at saturation by looking at the saturated mixing ratio calculated in Fig. 4. We can therefore expect condensation to occur during the night (and sublimation during the day as the diurnal cycle is observed to be approximately symmetrical) and an isotopic exchange between the lower atmospheric water vapor and the surface snow. This raises the question of whether, and by what magnitude, condensation and sublimation processes might affect the surface snow and lower the water vapor isotopic composition.

In order to address this question, we set up a simple box model as a closed system containing two interacting and homogeneous reservoirs. Figure 5 depicts the schematics of this model and introduces our notations. As this system is closed, the variation of moisture in the air (and its isotopic composition) is only due to condensation/sublimation during the 

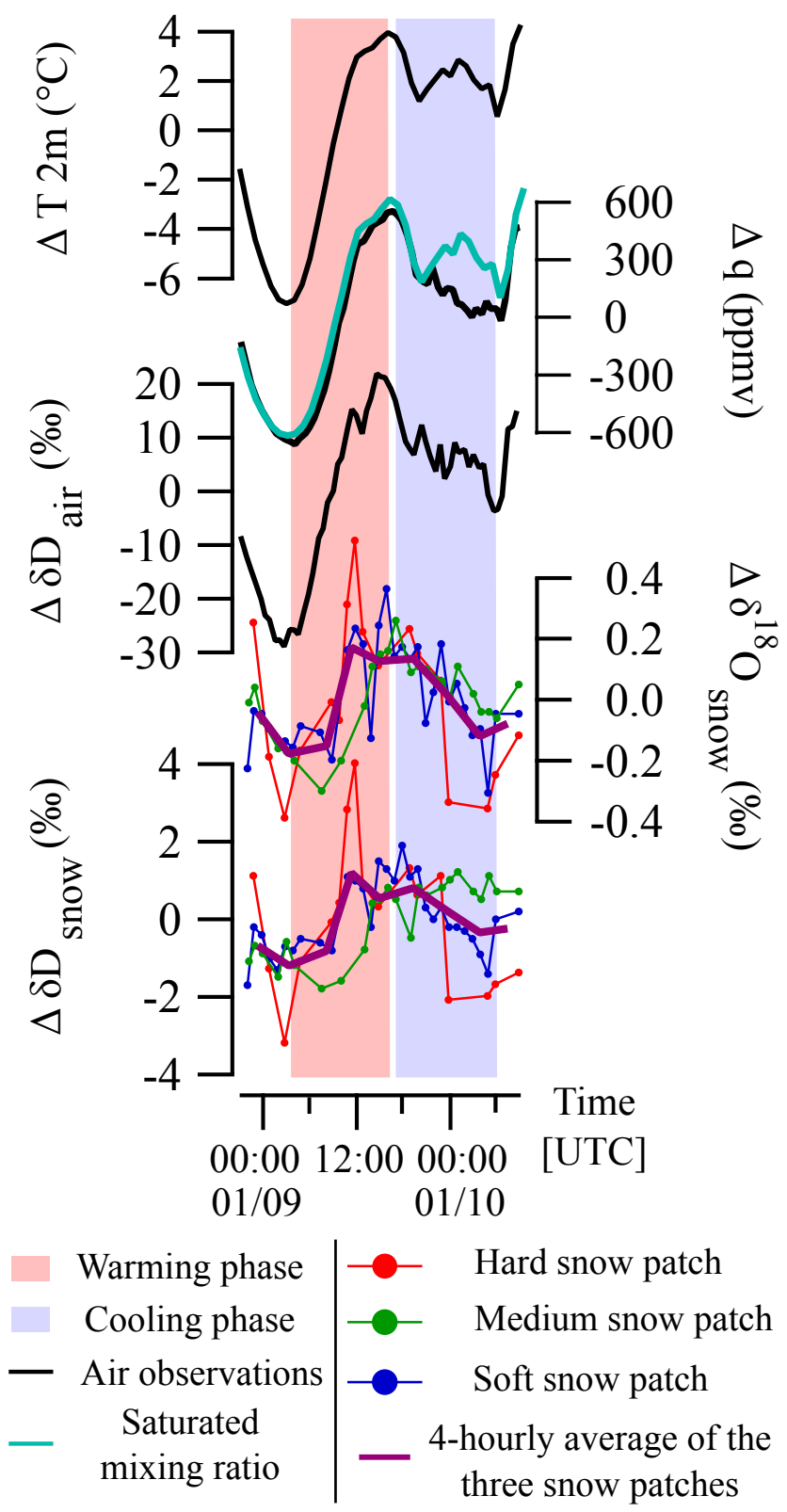

Figure 4. Observations performed during the Snow Surface Diurnal Cycle (SSDC) experiment from the 9 to 10 January 2014. The three different snow patches are labeled "hard", "medium" and "soft" according to their texture. Each hourly data point is the average of the measurements made on 5 snow surface samples per patch.

cooling/warming phase and we have mass conservation of the water molecules: $\forall t, m_{t}^{\mathrm{v}}+m_{t}^{\mathrm{s}}=m_{0}^{\mathrm{v}}+m_{0}^{\mathrm{s}}$ with $t=0$ the start of the cooling phase and " $\mathrm{v}$ " and "s" indices representing vapor and snow, respectively. Our simulation is based on the values from the mean stack of the 18 diurnal cycles instead of the specific day corresponding to the snow sampling because of the unusually high temperature and humidity during the night of 9 January 2014. We split our analysis into two parts: the cooling phase (from $\sim$ 17:00 to $\sim$ 02:00 UTC) and

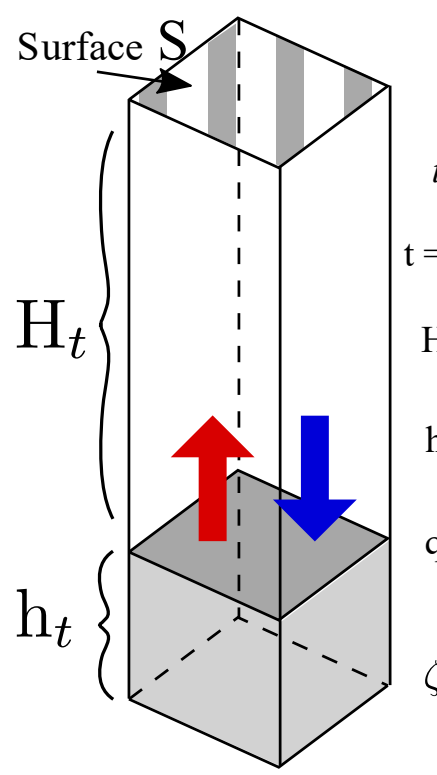

Box model - closed system -

$t$ : Hourly time step $=0$. Start of the cooling phase

$\mathrm{H}_{\mathrm{t}}$ : Height of the humid air column

$h_{t}$ : Height of the snow surface reservoir

$\mathrm{q}_{\mathrm{t}}$ : Humidity mixing-ratio measured at $3.0 \mathrm{~m}$

Surface height anomaly $\zeta_{t}:$ due to sublimation and condensation $\mathrm{m}_{t}^{v}, \mathrm{~m}_{t}^{s}$ : Water vapor mass and snow mass

$$
\begin{array}{c|c}
\mathrm{h}_{t}=\mathrm{h}_{0}+\zeta_{t} & \mathrm{~m}_{t}^{s}=\rho_{s} \mathrm{~h}_{t} \mathrm{~S} \\
\mathrm{H}_{t}=\mathrm{H}_{0}-\zeta_{t} & \mathrm{~m}_{t}^{v}=\mathrm{q}_{t} \rho_{d} \mathrm{H}_{t} \mathrm{~S}
\end{array}
$$

Snow accumulation during condensation:

$$
\zeta_{t+1} \approx \zeta_{t}+\frac{\rho_{d}}{\rho_{s}} \mathrm{H}_{0}\left(\mathrm{q}_{t}-\mathrm{q}_{t+1}\right)
$$

Order of magnitude of the parameters:

$$
\begin{array}{c|c}
\rho_{d}=0.95 \mathrm{~kg} \mathrm{~m}^{-3} & \mathrm{~h}_{0} \approx 2-5 \times 10^{-3} \mathrm{~m} \\
\rho_{s}=300-380 \mathrm{~kg} \mathrm{~m}^{-3} & \mathrm{q}_{t} \in 5-9 \times 10^{-4} \mathrm{~kg} \mathrm{~kg}^{-1} \\
\mathrm{H}_{0} \approx 50-100 \mathrm{~m} & \zeta^{\max } \approx 1.1-1.4 \times 10^{-4} \mathrm{~m}
\end{array}
$$

Figure 5. Schematic of the box model and description of the input parameters (isotopes excluded). $\zeta^{\max }$ is the maximum value of the surface height anomaly at the end of the cooling phase, calculated with two extreme values of $\left(H_{0}, \rho_{\mathrm{S}}\right)$. The height of the SSDC snow samples gives an estimation of $h_{0} \cdot \rho_{\mathrm{d}}$ and $\rho_{\mathrm{d}}$ have been calculated from 100 snow samples per day, surface pressure, temperature and relative humidity observed at Kohnen from December 2013 to January 2014.

the warming phase (from $\sim 03: 00$ to $\sim 15: 00$ UTC). More details concerning the equations present in the box model are given in the Supplement.

\subsubsection{Cooling phase}

The deposition of the condensate on the snow surface during the cooling phase is expected to reach a maximum height of $\zeta^{\max } \sim 0.1 \mathrm{~mm}$ (calculation detailed in Fig. 5). As the depth of our surface snow samples is $\sim 2-5 \mathrm{~mm}$, we mix the condensate with the snow reservoir. From $t$ to $t+1$, an amount 
$\left(m_{t}^{\mathrm{v}}-m_{t+1}^{\mathrm{v}}\right)$ condensates and the isotopic ratio of the condensate in equilibrium with the vapor is $\alpha_{t} R_{t}^{\mathrm{v}}$, where $R$ is the isotopic ratio of the heavy isotope and $\alpha_{t}$ the associated fractionation coefficient with respect to ice calculated from the air temperature measured at $2 \mathrm{~m}$ at time $t$. Assuming an immediate removal of the condensate from the air reservoir and an immediate mixing with the snow reservoir, we obtain the following for the isotopic composition of the vapor $\delta_{t}^{\mathrm{V}}$ and the isotopic composition of the snow $\delta_{t}^{\mathrm{s}}$ :

$\delta_{t+1}^{\mathrm{v}}+1000=A_{t}^{\mathrm{v}}\left(\delta_{t}^{\mathrm{v}}+1000\right)$,

$\delta_{t+1}^{\mathrm{s}}+1000=B_{t}^{\mathrm{s}}\left(\delta_{t}^{\mathrm{s}}+1000\right)+B_{t}^{\mathrm{v}}\left(\delta_{t}^{\mathrm{v}}+1000\right)$,

with

$A_{t}^{\mathrm{v}}=\frac{q_{t}}{q_{t+1}}-\alpha_{t}\left(\frac{q_{t}}{q_{t+1}}-1\right)$

$B_{t}^{\mathrm{s}}=\frac{\rho_{\mathrm{s}} h_{0}+\rho_{\mathrm{d}} H_{0}\left(q_{0}-q_{t}\right)}{\rho_{\mathrm{s}} h_{0}+\rho_{\mathrm{d}} H_{0}\left(q_{0}-q_{t+1}\right)}$

$B_{t}^{\mathrm{v}}=\alpha_{t} \frac{\rho_{\mathrm{d}} H_{0}\left(q_{t}-q_{t+1}\right)}{\rho_{\mathrm{s}} h_{0}+\rho_{\mathrm{d}} H_{0}\left(q_{0}-q_{t+1}\right)}$.

We will consider the equilibrium case (RH set to 1 ) as well as the supersaturated case $(\mathrm{RH}=1.1)$ by replacing $\alpha_{t}$ with the equivalent fractionation coefficient (Jouzel and Merlivat, 1984), which takes into account the kinetic effects in a supersaturated environment. The required input parameters for simulating $\delta_{t}^{\mathrm{v}}$ are $q_{t}, \delta_{0}^{\mathrm{v}}, \alpha_{t}$ and RH (set to 1 or 1.1). The required input parameters for simulating $\delta_{t}^{\mathrm{s}}$ are $q_{t}, \delta_{t}^{\mathrm{v}}$ (measured, not simulated), $\alpha_{t}, h_{0}, H_{0}, \mathrm{RH}, \rho_{\mathrm{s}}, \rho_{\mathrm{d}}$ and $\delta_{0}^{\mathrm{s}}$.

Figure 6 presents the simulation of $\delta \mathrm{D}_{t}^{\mathrm{V}}$ during the cooling phase, based on Eq. (2) and the isotopic variation of the condensate (Eq. (3) with $h_{0}=0 \mathrm{~mm}$ ), which does not depend on $H_{0}, \rho_{\mathrm{s}}$ or $\rho_{\mathrm{d}}$. We have used fractionation coefficients with respect to ice given by Merlivat and Nief (1967) and Ellehoj et al. (2013).

The amplitude of the simulated isotopic composition of the vapor is for each case 3 times larger than observed. The box model is closed, so any change in the vapor is forced to condensate in order to keep the mass conservation equation. In reality, there is advection of air masses with different moisture or temperature into and out of the box. Exchanges with the free troposphere are also possible. This could partly contribute to the decrease of humidity during the cooling phase in an open system instead of a pure condensation process in a closed system. Nevertheless, our simplistic approach leads to the conclusion that about $40 \%$ of the diurnal vapor mixing ratio variation is sufficient to simulate the right order of magnitude of isotopic variations, based on equilibrium fractionation. This is consistent with the results of ECHAM5-wiso: while this atmospheric model underestimates the diurnal variability of humidity (by $40 \%$ ), it does correctly capture the diurnal variability of deuterium.

The isotopic variation of the condensate is $\sim 6 \%$ and decreases in phase with the vapor. We define $\left(\delta \mathrm{D}_{0}^{\mathrm{s}}\right)_{\text {eq }}$ as the deuterium value of the condensate at equilibrium with the initial
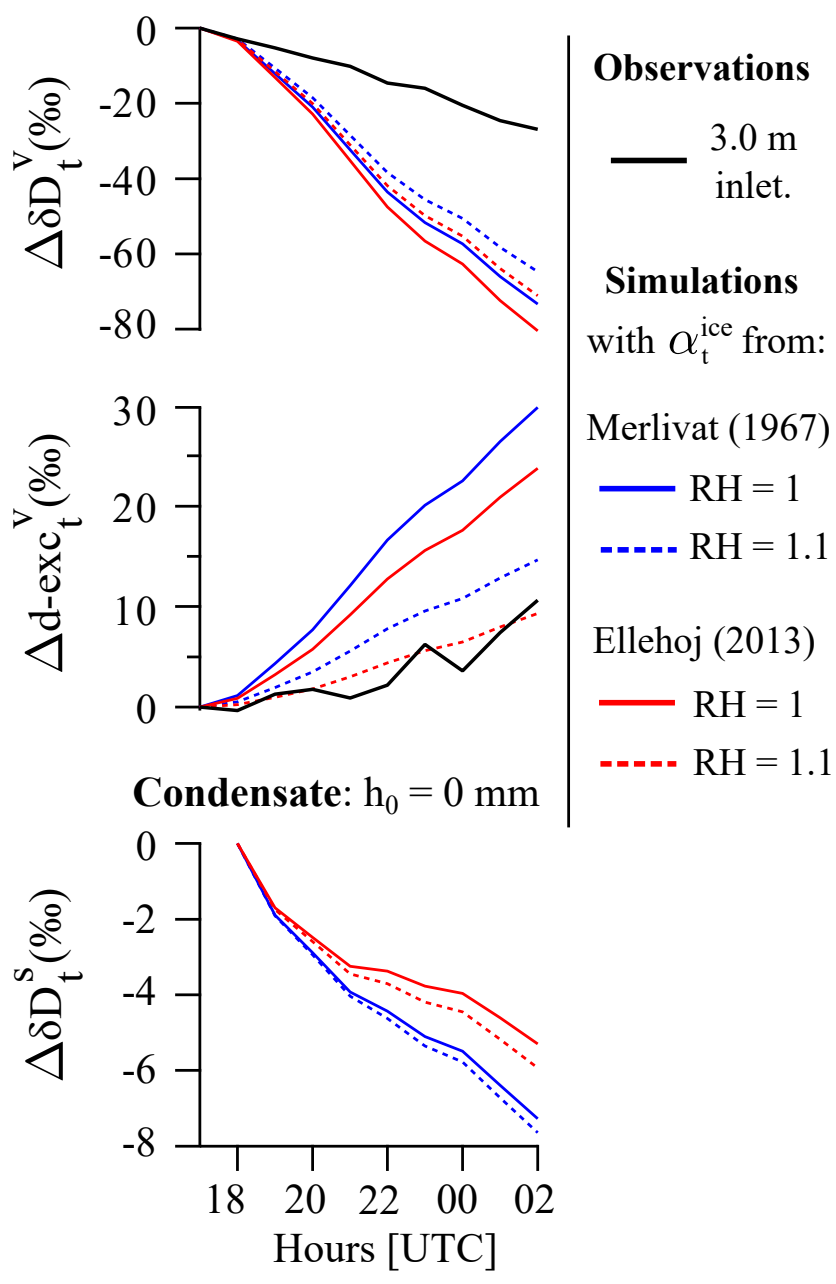

Figure 6. Measured and simulated (box model) deuterium anomalies of the vapor and simulated deuterium anomaly of the condensate $\left(h_{0}=0 \mathrm{~mm}\right)$ during the cooling phase, from 17:00 to 02:00 UTC. Observations come from the stack of the 18 diurnal cycles. Equivalent fractionation coefficients have been calculated from Jouzel and Merlivat (1984) for RH=1.1.

vapor. The value of $\left(\delta \mathrm{D}_{0}^{\mathrm{s}}\right)_{\text {eq }}$ is $-295 \%$ o using the fractionation coefficient from Merlivat and Nief (1967). This result is consistent with the mean value of the isotopic composition of the three snow patches (Table 6).

Figure 7 presents the simulation of $\delta \mathrm{D}_{t}^{\mathrm{s}}$ during the cooling phase, based on Eq. (3). We have used $\rho_{\mathrm{d}}=0.95 \mathrm{~kg} \mathrm{~m}^{-3}$, calculated from the surface pressure, temperature and $\mathrm{RH}$ measured at Kohnen, and $\rho_{\mathrm{s}}=340 \mathrm{~kg} \mathrm{~m}^{-3}$, calculated from 100 daily snow samples collected at Kohnen during the period of air measurement. The initial depth of the snow reservoir $h_{0}$ corresponds to the depth of the SSDC snow samples, i.e., between 2 and $5 \mathrm{~mm}$. The air reservoir is considered as the part of the lower atmosphere affected by convection and turbulence within a timescale of about $1 \mathrm{~h}$. The parameter $H_{t}$ is therefore the mixing-layer height. Sodar measurements performed at Dome C (Antarctica) showed magnitudes be- 


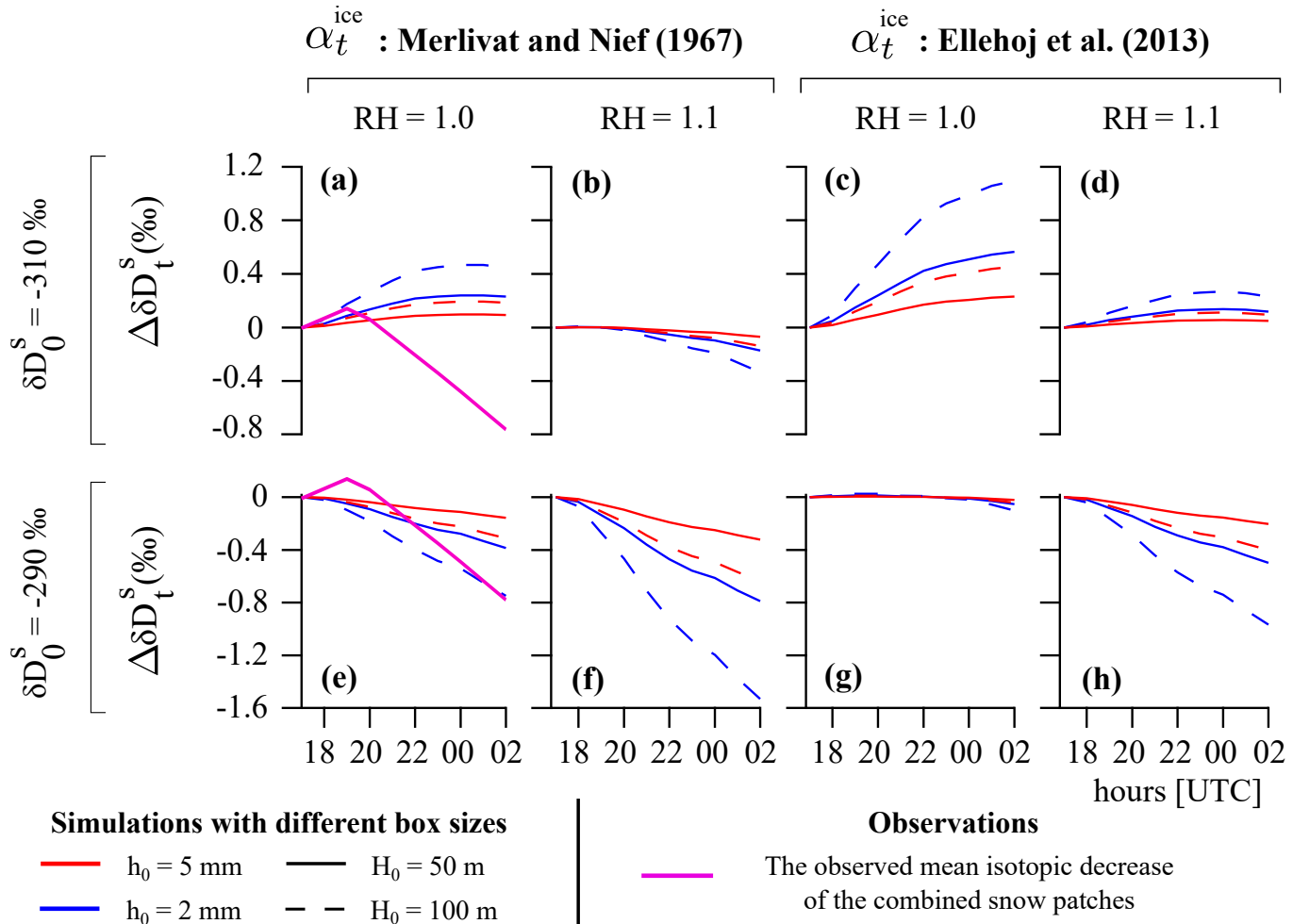

Figure 7. Simulated (box model) deuterium anomalies of the snow surface during the cooling phase, with $h_{0}, H_{0}, \mathrm{RH}, \alpha_{t}$ and $\delta \mathrm{D}_{0}^{\mathrm{S}}$ as varying inputs parameters. Fractionation coefficients at equilibrium come from Merlivat and Nief (1967) and Ellehoj et al. (2013), calculated with $T$ at $2 \mathrm{~m}$. Equivalent fractionation coefficients have been calculated from Jouzel and Merlivat (1984) for RH=1.1.

tween 10 and $300 \mathrm{~m}$ (Pietroni et al., 2012; Casasanta et al., 2014). In this study, we set the mixing-layer height as a constant and consider two cases: $H_{0}=50 \mathrm{~m}$ or $H_{0}=100 \mathrm{~m}$.

This simple parametrization allows us to test the sensitivity of the box model to the variations of $H_{t}$. We also have chosen two initial isotopic compositions of the snow surface as two distinct cases: (i) $\delta \mathrm{D}_{0}^{\mathrm{s}}=-310 \%$, below $\left(\delta \mathrm{D}_{0}^{\mathrm{s}}\right)_{\mathrm{eq}}$, and (ii) $\delta \mathrm{D}_{0}^{\mathrm{s}}=-290 \%$, above $\left(\delta \mathrm{D}_{0}^{\mathrm{s}}\right)_{\text {eq. }}$. In the first case, the mixing between the condensate and the snow surface will tend toward the equilibrium through a positive trend; in the second case, a negative trend is predicted. This is due to the difference between the isotopic composition of the condensate and the snow surface, positive or negative at a given time $t$. The response of the model shown in Fig. 7 depends strongly on parameters that are not well constrained. These parameters are the box sizes (a snow reservoir with a depth above $1 \mathrm{~cm}$ will keep a constant isotopic composition), the fractionation coefficients (disagreement between Merlivat and Nief, 1967, and Ellehoj et al., 2013) or the value of $\delta \mathrm{D}_{0}^{\mathrm{v}}$, which could be measured with an accuracy of $11 \%$ only (see Table 2). However, we are able to conclude that the condensation of water vapor has an effect on the isotopic composition of the top $2 \mathrm{~mm}$ of the snow surface.

\subsubsection{Warming phase}

It is generally assumed that no fractionation occurs during sublimation. Using Greenland data, Steen-Larsen et al. (2011) and Landais et al. (2012) showed that on average the snow surface isotopes and the water vapor isotopes are in equilibrium, and they estimated that the value of the equilibrium factor lies between the fractionation coefficient $\alpha^{\text {ice }}$ with respect to ice (Merlivat and Nief, 1967; Ellehoj et al., 2013) and the fractionation coefficient $\alpha^{\text {water }}$ with respect to water (Majoube, 1971). In this study, we test different hypotheses to obtain a range of prediction of the isotopic variation in the vapor and the snow surface. From $t$ to $t+1$, an amount $\left(m_{t+1}^{\mathrm{v}}-m_{t}^{\mathrm{v}}\right)$ sublimates and the isotopic ratio $R_{t}^{\text {sub }}$ of the sublimate will be tested under three different assumptions: (i) no fractionation occurs and $R_{t}^{\mathrm{sub}}=R_{t}^{\mathrm{s}}$; (ii) the sublimate is formed in equilibrium with the snow and $R_{t}^{\mathrm{sub}}=R_{t}^{\mathrm{s}} / \alpha_{t}$; (iii) the kinetic effect due to subsaturation is taken into account and a thin layer of liquid water above the snow with the same isotopic composition is considered. Following Merlivat and Jouzel (1979) we have in case (iii)

$$
R_{t}^{\mathrm{sub}}=\frac{1-k}{1-\mathrm{RH}}\left(\frac{R_{t}^{\mathrm{s}}}{\alpha_{t}}-\mathrm{RH} \times R_{t}^{\mathrm{v}}\right),
$$



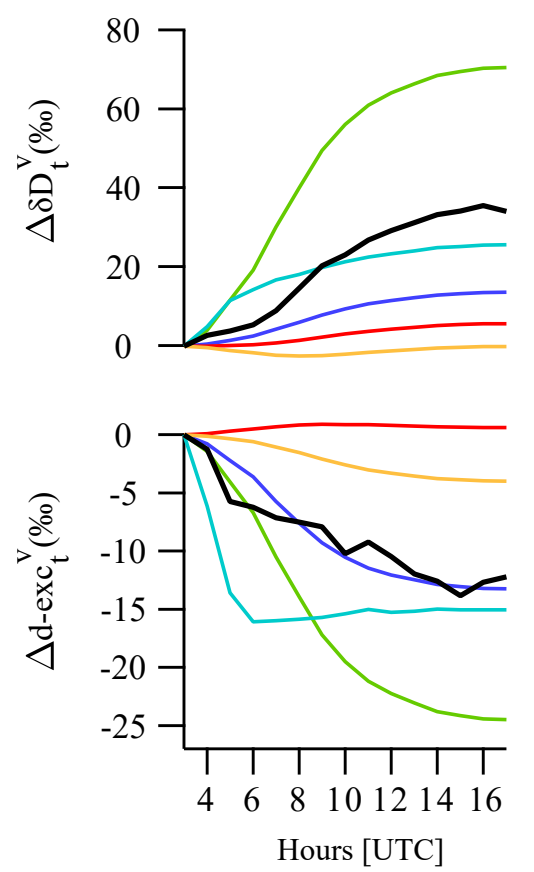

Observations

- $3.0 \mathrm{~m}$ inlet measurements

Simulations

$-\alpha_{t}=1$ (no fractionation)

- $\alpha_{t}^{\text {ice }}$ from (Merlivat an Nief, 1967)

- $\alpha_{t}^{\text {ice }}$ from (Ellehoj et al., 2013)

- $\alpha_{t}^{\text {water }}$ from (Majoube, 1971)

$-\alpha_{t}^{\text {water }}$ from (Majoube, 1971)

Taking into account the kinetic effect at subsaturation $(\mathrm{RH}=0.9)$

Figure 8. Measured and simulated (box model) deuterium and dexcess anomalies of the vapor during the warming phase. Kinetic effects occurring at subsaturation $(\mathrm{RH}=0.9)$ have been calculated following Merlivat and Jouzel (1979) in a smooth regime.

with the RH set equal to 0.9 , and the kinetic fractionation factor $k$ given by $k_{\delta^{18} \mathrm{O}}=6.2 \% \circ$ and $k_{\delta \mathrm{D}}=5.5 \%$. We present the equations for the vapor and the snow surface for the case (iii) only, noticing that cases (i) and (ii) are mathematically obtained from case (iii).

Assuming an immediate removal of the sublimate from the snow reservoir and an immediate mixing with the molecules contained in the air reservoir, we have for the isotopic composition of the vapor:

$\delta_{t+1}^{\mathrm{v}}+1000=E_{t}^{\mathrm{v}}\left(\delta_{t}^{\mathrm{v}}+1000\right)+E_{t}^{\mathrm{s}}\left(\delta_{t}^{\mathrm{s}}+1000\right)$,

with

$$
\begin{aligned}
& E_{t}^{\mathrm{v}}=\frac{q_{t}}{q_{t+1}}-\mathrm{RH} \times \frac{1-k}{1-\mathrm{RH}}\left(1-\frac{q_{t}}{q_{t+1}}\right) \\
& E_{t}^{\mathrm{s}}=\frac{1}{\alpha_{t}} \frac{1-k}{1-\mathrm{RH}}\left(1-\frac{q_{t}}{q_{t+1}}\right) .
\end{aligned}
$$

The equations for the isotopic composition of the snow are

$\delta_{t+1}^{\mathrm{s}}+1000=F_{t}^{\mathrm{s}}\left(\delta_{t}^{\mathrm{s}}+1000\right)+F_{t}^{\mathrm{v}}\left(\delta_{t}^{\mathrm{v}}+1000\right)$,

with

$$
\begin{aligned}
F_{t}^{\mathrm{s}} & =\frac{\rho_{\mathrm{s}} h_{0}+\rho_{\mathrm{d}} H_{0}\left(q_{0}-q_{t}-\frac{1}{\alpha_{t}} \frac{1-k}{1-\mathrm{RH}}\left(q_{t+1}-q_{t}\right)\right)}{\rho_{\mathrm{s}} h_{0}+\rho_{\mathrm{d}} H_{0}\left(q_{0}-q_{t+1}\right)} \\
F_{t}^{\mathrm{v}} & =\mathrm{RH} \times \frac{1-k}{1-\mathrm{RH}} \times \frac{\rho_{\mathrm{d}} H_{0}\left(q_{t+1}-q_{t}\right)}{\rho_{\mathrm{s}} h_{0}+\rho_{\mathrm{d}} H_{0}\left(q_{0}-q_{t+1}\right)} .
\end{aligned}
$$

Figure 8 displays the measurements and simulations performed for the isotopic composition of the deuterium and dexcess of the vapor during the warming phase. When no fractionation occurs during sublimation, the simulated variation of the deuterium is 2 times higher than observed. If we were sublimating a block of solid ice, it would be conceivable that only the very surface atoms would be able to sublimate, and the system would not fractionate. However, in the presence of very porous snow, there are a very large numbers of water molecules participating in the snow-air interface, and it is possible that snow would behave more like a liquid than like a solid in this respect and would fractionate. We tested for the presence of fractionation by running the box model with a variety of available fractionation factors for air over ice and water (Merlivat and Nief, 1967; Ellehoj et al., 2013; Majoube, 1971) and find that using $\alpha^{\text {ice }}$ the model underestimates the variations in $\delta \mathrm{D}$ (Fig. 8). The true fractionation factor at sublimation is probably lower than $\alpha^{\text {ice }}$, but the crude nature of our model prevents us from quantifying it precisely.

Figure 9 presents the simulated $\delta \mathrm{D}$ of the surface snow during the warming phase. We notice that $\delta_{t+1}^{\mathrm{s}}=\delta_{t}^{\mathrm{s}}$ when no fractionation occurs as it is assumed in ECHAM5-wiso and LMDZiso. A difference between equilibrium and subsaturation has to be noted in Eq. (5) due to the coefficient $F_{t}^{\mathrm{V}}$. If $F_{t}^{\mathrm{v}}=0$ (equilibrium), there is no influence of the vapor on the isotopic composition of the surface snow and $\delta_{0}^{\mathrm{s}}$ will not have a significant impact on $\delta_{t}^{\text {s }}$ : any patch of snow will share the same isotopic variation whatever its initial isotopic composition is. If $F_{t}^{\mathrm{v}} \neq 0$ (subsaturation), the isotopic composition of the snow is affected by the isotopic composition of the vapor, and in that case the variation of the isotopic composition of a snow patch during the warming phase will depend on its initial isotopic composition $\delta_{0}^{\mathrm{s}}$ (Fig. 7).

We focus here only on $\delta \mathrm{D}_{0}^{\mathrm{s}}=-320 \%$, which is the average of the 100 daily snow samples collected at Kohnen over the measurement period. Our data from the three snow patches consistently depict a positive trend during the warming phase, with an amplitude between 3 and $7 \%$ for $\Delta \delta \mathrm{D}$ 


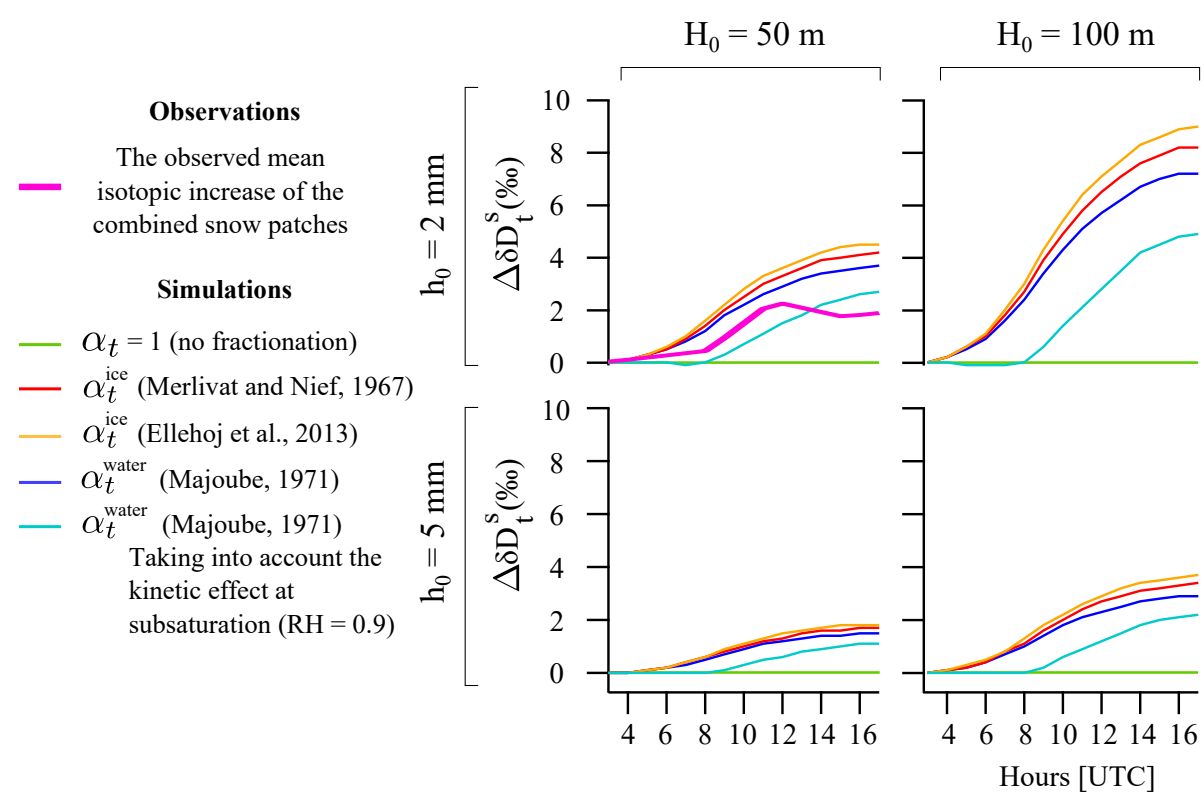

Figure 9. Simulated (box model) deuterium anomalies of the snow surface during the warming phase, with $h_{0}, H_{0}$ and the fractionation coefficients as varying inputs parameters. The initial isotopic composition of the snow surface has been taken as the average of the 100 daily snow samples collected at Kohnen over the period of measurements, $\delta \mathrm{D}_{0}^{\mathrm{S}}=-320 \%$.

(Fig. 9). Simulations with different values of $H_{0}, h_{0}$ and $\alpha_{t}$ share the same positive trend with a peak-to-peak amplitude between 1 and $8 \%$, which is of the same order of magnitude as the observations. We notice that the uncertainties related to the heights of the reservoirs have greater impacts on the simulated snow surface isotopic values than the different fractionation coefficients. As a result, we are not able to constrain the fractionation factor at sublimation, but we observe that since the surface snow isotopic composition is changing, the sublimation process must be associated with an isotopic fractionation.

\section{Conclusions}

Continuous measurements of temperature, humidity mixing ratio and water vapor isotopes were performed during summer 2013/2014 at Kohnen station in East Antarctica. These data highlight a strong diurnal cycle, in contrast to rather stable day-to-day mean levels over 1 month of observations. During our monitoring period, the surface vapor isotopic composition was therefore more driven by local processes than by synoptic changes. Outputs from the two AGCMs (ECHAM5-wiso and LMDZiso) show in general good agreements with the observations. However, the surface temperature variations simulated by the models have an amplitude $50 \%$ lower than observed by Van As et al. (2005), likely due to the difficulty to simulate the longwave radiative budget (related to the cloud cover and snowfall events). Moreover, the strong katabatic winds observed at Kohnen are not properly simulated by the AGCMs. The simulation of processes in the polar boundary layer and associated inversion is also known to be a challenge for AGCMs (Holtslag et al., 2013). This could explain why the amplitude of the diurnal cycles is lower in the models compared to the observations.

We have investigated the diurnal isotopic response of the upper thin layer of snow surface to the atmospheric variations. A continuous hourly sampling over $35 \mathrm{~h}$ of the first $\sim 2-5 \mathrm{~mm}$ of the snow surface of three different snow patches reveals a significant variability in both $\delta \mathrm{D}$ and $\delta^{18} \mathrm{O}$ during a period without snowfall events. As these variations in the surface snow isotopic composition follow the diurnal trend in the air, this result confirms the observations of Steen-Larsen et al. (2014) at NEEM who also observed parallel variations between the snow surface isotopic composition and lower atmosphere isotopic composition. These observations were, however, with a higher variation on a day-to-day scale. In their case, they reported larger variations in the parallel variations in the isotopic composition of both surface snow (5 mm) and vapor, reaching $10 \%$ over 5 days.

Two important consequences can be inferred from the snow sample diurnal observations: (1) post-depositional processes have a significant impact on the isotopic composition of the snow surface and (2) the sublimation process is fractionating. These two points are not included in classical isotopic theory and therefore not implemented in atmospheric models.

In order to determine the contributions of condensation and sublimation to the isotopic variations of the vapor and surface snow, we developed a simple model describing the isotopic exchange between two reservoirs contained in a 
closed system: a water vapor column and a thin snow surface layer. We find that the observed isotopic variations in the water vapor phase $\left(\delta \mathrm{D}^{\mathrm{v}}\right)$ are about half of what simple condensation / evaporation equilibrium would dictate. This is likely due to advection, exchanges with the free troposphere and variations in the boundary layer height. Additionally, for our observed isotopic composition of surface $(2-5 \mathrm{~mm}$ ) snow changes during both the warming and the cooling phase (peak-to-peak variation of $\sim 3 \%$ o for $\delta \mathrm{D}$ ), our crude model is able to reproduce these observations, although the model results depend strongly on the size of the reservoir chosen. According our box model, no diurnal cycle in the isotopic composition of the snow surface is expected from a depth of $1 \mathrm{~cm}$ or above. We do observe an increase in $\delta \mathrm{D}^{\mathrm{s}}$ during the sublimation process, which indicates that water isotopes undergo fractionation during sublimation. The only doubt we could emit on this result is related to the wind drift. Effectively, the isotopic variability observed on the diurnal scale in a snow patch could also be attributed to the renewal of the snow surface by the wind, which mixes the surface of the snow with ice crystals coming from other snow patches. Assuming that fractionation occurs during sublimation, the uncertainties in the model geometry and in air advection prevent us from being able to determine the fractionation coefficient. Our water vapor isotope data suggest, however, that it is smaller than $\alpha^{\text {ice }}$. Further analyses are required to quantify the impact of post-depositional processes on the isotopic signal from ice core data.

The day-to-day variations in water vapor isotopic composition have a much smaller amplitude than the diurnal cycle, because no large synoptic event was recorded during our monitoring period. Expanding the temporal framework of such monitoring is a pre-requisite in order to better understand the importance of horizontal advection and to evaluate the processes at play during the winter season. Our observations show the possible importance of surface snow-surface vapor exchanges for the isotopic composition recorded in ice cores. This not only stresses the potential of isotopic monitoring of snow-air interactions for the study of fractionation processes during water phase change but also underscore the importance of improvements in analytical accuracy under low humidity conditions. This constitutes an experimental challenge for future works.

\section{The Supplement related to this article is available online at doi:10.5194/tc-10-1647-2016-supplement.}

Acknowledgements. This first paper is dedicated to Dominique Hirondel, former teacher of history of sciences, for being a role model for scientific curiosity and fascination with reality. The scientific campaign would not have been possible without the efficient work of the AWI logistic staff. Special thanks to Carleen Reijmer for providing the data of AWS9, to Michael Schaefer, Gert Koenig-Langlo and Bernd Loose for their contribution to the meteorological observations and to Max Berkelhammer for his valuable contribution to the final version of the manuscript. We also are grateful for the detailed and constructive comments offers by one anonymous reviewer and Bradley Markle, which improved the paper significantly. LMDZiso simulations were performed on the Ada supercomputer at IDRIS under GENCI project 0292. ECHAM5-wiso simulations were performed on AWI's high-performance computing systems with support from the Scientific Computing research group.

Edited by: M. van den Broeke

Reviewed by: B. Markle and one anonymous referee

\section{References}

Anderson, P.: A method for rescaling humidity sensors at temperatures well below freezing, J. Atmos. Ocean. Tech., 11, 13881391, 1994.

Angert, A., Lee, J.-E., and Yakir, D.: Seasonal variations in the isotopic composition of near-surface water vapour in the eastern Mediterranean, Tellus B, 60, 674-684, 2008.

Baer, D. S., Paul, J. B., Gupta, M., and O'Keefe, A.: Sensitive absorption measurements in the near-infrared region using off-axis integrated cavity output spectroscopy, Proc. SPIE 4817, Diode Lasers and Applications in Atmospheric Sensing, 24 September 2002, 167 pp., doi:10.1117/12.451461, 2002.

Berrisford, P., Dee, D., Poli, P., Brugge, R., Fielding, K., Fuentes, M., Kållberg, P., Kobayashi, S., Uppala, S., and Simmons, A.: The ERA-Interim archive Version 2.0, Shinfield Park, Reading, 2011.

Birnbaum, G., Freitag, J., Brauner, R., König-Langlo, G., Schulz, E., Kipfstuhl, S., Oerter, H., Reijmer, C. H., Schlosser, E., Faria, S. H., Ries, H., Loose, B., Herber, A., Duda, M. G., Powers, J. G., Manning, K. W., and Van den Broeke, M. R.: Strong-wind events and their influence on the formation of snow dunes: observations from Kohnen station, Dronning Maud Land, Antarctica, J. Glaciol., 56, 891-902, 2010.

Casasanta, G., Pietroni, I., Petenko, I., and Argentini, S.: Observed and modelled convective mixing-layer height at Dome C, Antarctica, Bound.-Lay. Meteorol., 151, 597-608, 2014.

Dansgaard, W.: Stable isotopes in precipitation, Tellus A, 16, 436468, 1964.

Ekaykin, A. A., Lipenkov, V. Y., Barkov, N. I., Petit, J. R., and Masson-Delmotte, V.: Spatial and temporal variability in isotope composition of recent snow in the vicinity of Vostok station, Antarctica: implications for ice-core record interpretation, Ann. Glaciol., 35, 181-186, 2002.

Ellehoj, M., Steen-Larsen, H. C., Johnsen, S. J., and Madsen, M. B.: Ice-vapor equilibrium fractionation factor of hydrogen and oxygen isotopes: Experimental investigations and implications for stable water isotope studies, Rapid Commun. Mass Spectrom., 27, 2149-2158, 2013.

Fujita, K. and Abe, O.: Stable isotopes in daily precipitation at Dome Fuji, East Antarctica, Geophys. Res. Lett. 33, 118503, doi:10.1029/2006GL026936, 2006.

Geldern, R. and Barth, J. A.: Optimization of instrument setup and post-run corrections for oxygen and hydrogen stable iso- 
tope measurements of water by isotope ratio infrared spectroscopy (IRIS), Limnol. Oceanogr. Meth., 10, 1024-1036, 2012. Holtslag, A., Svensson, G., Baas, P., Basu, S., Beare, B., Beljaars, A., Bosveld, F., Cuxart, J., Lindvall, J., Steeneveld, G., Tjernström, M., and Van De Wiel, B. J. H.: Stable atmospheric boundary layers and diurnal cycles: challenges for weather and climate models, B. Am. Meteorol. Soc., 94, 1691-1706, 2013.

Jacob, H. and Sonntag, C.: An 8-year record of the seasonal variation of ${ }^{2} \mathrm{H}$ and ${ }^{18} \mathrm{O}$ in atmospheric water vapour and precipitation at Heidelberg, Germany, Tellus B, 43, 291-300, doi:10.1034/j.1600-0889.1991.t01-2-00003.x, 1991.

Joussaume, S., Sadourny, R., and Jouzel, J.: A general circulation model of water isotope cycles in the atmosphere, Nature, 311, 24-29, doi:10.1038/311024a0, 1984.

Jouzel, J. and Merlivat, L.: Deuterium and oxygen 18 in precipitation: Modeling of the isotopic effects during snow formation, $\mathrm{J}$. Geophys. Res.-Atmos., 89, 11749-11757, 1984.

Landais, A., Steen-Larsen, H. C., Guillevic, M., Masson-Delmotte, V., Vinther, B., and Winkler, R.: Triple isotopic composition of oxygen in surface snow and water vapor at NEEM (greenland), Geochim. Cosmochim. Ac., 77, 304-316, 2012.

Majoube, M.: Fractionnement en oxygene-18 et en deuterium entre l'eau et sa vapeur, J. Chim. Phys., 68, 1423-1436, 1971.

Masson-Delmotte, V., Hou, S., Ekaykin, A., Jouzel, J., Aristarain, A., Bernardo, R., Bromwich, D., Cattani, O., Delmotte, M., Falourd, S., Frezzotti, M., Gallée, H., Genoni, L., Isaksson, E., Landais, A., Helsen, M., Hoffmann, G., Lopez, J., Morgan, V., Motoyama, H., Noone, D., Oerter, H., Petit, J., Royer, R., Uemura, R., Schmidt, G., Schlosser, E., Simões, J., Steig, E., Stenni, B., Stievenard, M., van den Broeke, M., van de Wal, R., van de Berg, W., Vimeux, F., and White, J.: A review of Antarctic surface snow isotopic composition: Observations, atmospheric circulation, and isotopic modeling, J. Climate, 21, 3359-3387, 2008.

Merlivat, L. and Jouzel, J.: Global climatic interpretation of the deuterium-oxygen 18 relationship for precipitation, J. Geophys. Res., 84, 5029-5033, 1979.

Merlivat, L. and Nief, G.: Fractionnement isotopique lors des changements d'état solide-vapeur et liquide-vapeur de l'eau à des températures inférieures à $0^{\circ} \mathrm{C}$, Tellus A, 19, 122-127, 1967.

Pietroni, I., Argentini, S., Petenko, I., and Sozzi, R.: Measurements and parametrizations of the atmospheric boundary-layer height at Dome C, Antarctica, Bound.-Lay. Meteorol., 143, 189-206, 2012.

Rast, S., Brokopf, R., Cheedela, S.-K., Esch, M., Gayler, V., Kirchner, I., Kornbluh, L., Rhodin, A., Schmidt, H., Schulzweida, U., and Wieners, K.-H.: User manual for ECHAM6, 21 June 2013, version echam-6.1.06p3-guide1.3.Hamburg, Max-Planck-Institut für Meteorologie Berichte zur Erdsystemforschung, 220 pp., doi:10.17617/2.1810486, 2013.
Risi, C., Bony, S., Vimeux, F., and Jouzel, J.: Water-stable isotopes in the LMDZ4 general circulation model: Model evaluation for present-day and past climates and applications to climatic interpretations of tropical isotopic records, J. Geophys. Res.-Atmos., 115, D12118, doi:10.1029/2009JD013255, 2010.

Roeckner, E., Bäuml, G., Bonaventura, L., Brokopf, R., Esch, M., Giorgetta, M., Hagemann, S., Kirchner, I., Kornblueh, L., Manzini, E., and Rhodin, A.: The atmospheric general circulation model ECHAM 5. PART I: Model description, Report, MPI für Meteorologie, 349 pp., 2003.

Schlosser, E., Reijmer, C., Oerter, H., and Graf, W.: The influence of precipitation origin on the $\delta^{18} \mathrm{O}-T$ relationship at Neumayer Station, Ekströmisen, Antarctica, Ann. Glaciol., 39, 41-48, 2004.

Schlosser, E., Manning, K., Powers, J., Duda, M., Birnbaum, G., and Fujita, K.: Characteristics of high-precipitation events in Dronning Maud Land, Antarctica, J. Geophys. Res.-Atmos., 115, D14107, doi:10.1029/2009JD013410, 2010.

Schwarz, G., Kowski, P., and Gernandt, H.: Saisonale Variation von Deuterium und Sauerstoff-18 in Luftfeuchte und Niederschlag über Antarktika, Isotop. Environ. Health Stud., 34, 159-168, doi:10.1080/10256019708036343, 1998.

Steen-Larsen, H. C., Masson-Delmotte, V., Sjolte, J., Johnsen, S. J., Vinther, B. M., Bréon, F.-M., Clausen, H., Dahl-Jensen, D., Falourd, S., Fettweis, X., and Gallée, H.: Understanding the climatic signal in the water stable isotope records from the NEEM shallow firn/ice cores in northwest Greenland, J. Geophys. Res.Atmos., 116, D06108, doi:10.1029/2010JD014311, 2011.

Steen-Larsen, H. C., Johnsen, S. J., Masson-Delmotte, V., Stenni, B., Risi, C., Sodemann, H., Balslev-Clausen, D., Blunier, T., Dahl-Jensen, D., Ellehøj, M. D., Falourd, S., Grindsted, A., Gkinis, V., Jouzel, J., Popp, T., Sheldon, S., Simonsen, S. B., Sjolte, J., Steffensen, J. P., Sperlich, P., Sveinbjörnsdóttir, A. E., Vinther, B. M., and White, J. W. C.: Continuous monitoring of summer surface water vapor isotopic composition above the Greenland Ice Sheet, Atmos. Chem. Phys., 13, 4815-4828, doi:10.5194/acp-13-4815-2013, 2013.

Steen-Larsen, H. C., Masson-Delmotte, V., Hirabayashi, M., Winkler, R., Satow, K., Prié, F., Bayou, N., Brun, E., Cuffey, K. M., Dahl-Jensen, D., Dumont, M., Guillevic, M., Kipfstuhl, S., Landais, A., Popp, T., Risi, C., Steffen, K., Stenni, B., and Sveinbjörnsdottír, A. E.: What controls the isotopic composition of Greenland surface snow?, Clim. Past, 10, 377-392, doi:10.5194/cp-10-377-2014, 2014.

Van As, D., Van Den Broeke, M., and Van De Wal, R.: Daily cycle of the surface layer and energy balance on the high Antarctic Plateau, Antarct. Sci., 17, 121-133, 2005.

Werner, M., Langebroek, P. M., Carlsen, T., Herold, M., and Lohmann, G.: Stable water isotopes in the ECHAM5 general circulation model: Toward high-resolution isotope modeling on a global scale, J. Geophys. Res.-Atmos., 116, d15109, doi:10.1029/2011JD015681, 2011. 